\title{
Optimum Policies for an Energy Harvesting Transmitter Under Energy Storage Losses
}

\author{
Kaya Tutuncuoglu, Student Member, IEEE, Aylin Yener, Fellow, IEEE, and Sennur Ulukus, Member, IEEE
}

\begin{abstract}
We consider an energy harvesting network where the transmitter harvests energy from nature, and the harvested energy can be saved in an imperfect battery which suffers from charging/discharging inefficiency. In particular, when $E$ units of energy is to be stored in the battery, only $\eta E$ units is saved and $(1-\eta) E$ is lost due to charging/discharging inefficiency, where $0 \leq \eta \leq 1$ represents the storing efficiency. We determine the optimum offline transmit power schedule for such a system for single-user and broadcast channel models, for static and fading channels, with and without a finite battery size. We show that the optimum policy is a double-threshold policy: specifically, we store energy in the battery only when the harvested energy is above an upper threshold, and retrieve energy from the battery only when the harvested energy is below a lower threshold; when the harvested energy is in between these two thresholds, we use it in its entirety in the current slot. We show that the two thresholds remain constant unless the battery is depleted or full. We provide an algorithm to determine the sequence of optimum thresholds. For the case with fading, we develop a directional water-filling algorithm which has a double-threshold structure. Finally, we formulate the online problem using dynamic programming, and numerically observe that the online policy exhibits a doublethreshold structure as well.
\end{abstract}

Index Terms-Energy harvesting communications, optimal packet scheduling, nodes with rechargeable batteries, inefficient energy storage.

\section{INTRODUCTION}

We consider an energy harvesting network where the transmitter harvests energy from nature to sustain its operation. In particular, the transmitter uses the energy harvested from nature to transmit its data packets. Such energy harvesting capabilities bring new constraints into the communication problem in the physical layer in the form of energy causality and noenergy-overflow conditions. The first constraint imposes that the energy that has not yet been harvested cannot be used for communication, and the second constraint imposes that no harvested energy should be allowed to overflow due to a finite battery size. Therefore, in such energy harvesting systems, packet scheduling and the corresponding energy management

Manuscript received April 1, 2014; revised September 10, 2014 and November 26, 2014; accepted December 4, 2014. The associate editor coordinating the review of this paper was L. Milstein.

This work was supported by NSF Grants CNS 09-64364/CNS 09-64632 and CCF $14-22347 /$ CCF $14-22111$. This paper was presented in part at the 46th Conference on Information Sciences and Systems, and the 2012 Asilomar Conference on Signals, Systems, and Computers.

K. Tutuncuoglu and A. Yener are with the department of Electrical Engineering, Pennsylvania State University, University Park, PA 16802 USA (e-mail: kaya@psu.edu; yener@ee.psu.edu).

S. Ulukus is with the Department of Electrical and Computer Engineering, University of Maryland, College Park, MD 20742 USA (e-mail: ulukus@umd.edu).

Digital Object Identifier scheme must be carefully optimized in the physical layer in order to guarantee a certain optimum performance.

Energy constrained communication was studied widely in the literature [1]-[6], including finite horizon scenarios with delay constraints [7]-[10]. The offline energy management problem in an energy harvesting setting was first formulated in [11], which considered the problem of minimizing the transmission completion time for a given number of data packets in an offline setting. This reference introduced the energy causality constraint, and showed that the transmitter should use as constant power as possible, subject to energy causality imposed by the energy harvesting profile. For a finitesized battery, [12] formulated the throughput maximization problem in a similar communication setting. Reference [12] introduced the no-energy-overflow constraint due to the finitesized battery, and showed that the transmitter should use as constant power as possible subject to energy causality and no-energy-overflow constraints, particularly, using harvested energy slow enough not to violate energy causality but fast enough to open up space in the finite-sized battery and cause no energy overflows. Reference [13] considered a fading channel and developed a directional water-filling algorithm where energy (water) is filled over the fading profile, with a directional flow of water to the right only, due to energy causality constraints: energy can be saved and used in the future, but the energy that will be harvested in the future cannot be used earlier; see also [14] for a treatment of the fading case. This line of work has been extended for broadcast channels in [15]-[17], multiple access channels in [18], interference channels in [19], two-hop relay channels in [20]-[26]. The effects of circuit power have been considered in [27]-[30], where the transmitter incurs energy loss by being on, i.e., when the transmit power is non-zero. This, then, disfavors long and constant stretches of transmit powers as this increases circuit energy consumption. Reference [27] shows the optimality of a directional glue-pouring algorithm in this case. This line of offline energy management has also been extended to energy cooperation in [31] where users transfer energy to one another, leading to two-dimensional direction water-filling in two-way and multiple access channels; see also bidirectional cooperation in [32], [33]. Receiver side energy harvesting has been considered in [34], [35]. Common in all of these works is the assumption that the energy harvesting nodes have perfect energy storage units (batteries) into which energy can be stored without any losses and in which energy can be saved without any leakages until it is eventually used.

In this paper, we consider energy storage imperfections in the form of charging/discharging inefficiency and their 
effects on the offline throughput maximization problem. Energy storage/retrieval imperfections can manifest themselves in many different ways [36], [37], for instance: imperfections in energy conversion from one technology to another, charging/discharging imperfections (only a portion of the available energy can be saved in the battery at the time of charging), energy leakage over time (saved energy is leaked and lost over time), battery size degradation (battery capacity size gets smaller at every recharge), etc. The first work to formulate a form of practical energy storage inefficiency in the context of offline throughput maximization is [38]. In [38], two forms of storage imperfections are considered: leakage of saved energy over time and battery degradation. The major effect of such imperfections on the throughput maximization problem is that they modify the energy feasibility tunnel, which is the tunnel that is formed by the energy causality upper staircase and the no-energy-overflow lower staircase [12]. The distance between these two staircases is exactly the size of the battery, $E_{\max }$, in the case of perfect storage [12]. Reference [38] demonstrated that, in the cases of energy leakage and battery degradation, the energy feasibility tunnel gets narrower by upper staircase decreasing and lower staircase increasing over time, and developed the optimum offline power allocation policy that maximizes the throughput.

The imperfections studied in [38] are long-term effects on energy storage, that affect communications in durations much larger than typical symbol durations. In this paper, we study another class of energy storage inefficiencies, which occur at the time of energy storage, almost instantaneously, at much shorter time durations. In particular, we consider the inefficiency (loss) that occurs at the time of charging/discharging ${ }^{1}$ : when $E$ units of energy is to be stored in the battery, only $\eta E$ units is saved and $(1-\eta) E$ is lost instantaneously due to charging/discharging inefficiency, where $0 \leq \eta \leq 1$ represents the storage efficiency. Depending on the technology used in energy storage, $\eta$ can be as low as 66\% [36], [37], [39], [40]. Such losses have been considered in communications in [41], [42] for duty-cycling with constant transmission rate under energy neutrality conditions, but not in the context of offline throughput maximization. In this paper, we consider the offline throughput maximization under such losses and determine the corresponding optimum energy management policies. We start with a single-user Gaussian channel with infinite-sized battery (Section III), then consider a finite-sized battery (Section IV), extend to fading channels (Section V), and extend to a multiuser broadcast setting (Section VI).

The effects of imperfections at charging/discharging considered in this paper are significantly different than leakage/degradation imperfections studied in [38]. In particular, while leakage/degradation imperfections affect the shape of the energy feasibility tunnel, in our case, the energy feasibility tunnel is unaffected. Instead, in our case, we need to determine, what portion of the incoming energy to store despite storage losses, and how to use the stored energy. We show

\footnotetext{
${ }^{1}$ While we consider both inefficiencies at the time of charging/discharging, we show that, from a mathematical point of view, these two imperfections can be clubbed together into a single effective inefficiency only at the time of charging.
}

that the optimal power policy has a double-threshold structure: whenever the harvested energy is below a lower threshold, we use a constant transmit power equal to that threshold by retrieving energy from the battery; whenever the harvested energy is above an upper threshold, we use a constant transmit power equal to that threshold by storing some of the harvested energy; and whenever the harvested energy is between these two thresholds, we use the harvested energy for transmission in its entirety without storing any of it in the battery. It then only remains to determine these thresholds. These thresholds change throughout the communication session and depend on the harvested energy profile and the storage efficiency $\eta$. We identify the properties these threshold should satisfy, and then provide an algorithm to determine these thresholds. In particular, we show that the optimal thresholds are constant between battery events, i.e., they change only when the battery is depleted or the battery is full, and may only increase if the battery is empty and may only decrease if the battery is full. In the case of a fading channel, we develop a modified version of directional water-filling which takes this double-threshold policy into account. For the broadcast channel, we determine the largest throughput region by employing double-threshold policies on weighted sum rate maximization problems.

Overall, we observe that contrary to the results of previous work with ideal batteries [11]-[17], where the optimal policies were shown to be piecewise constant, here, the optimal policy may favor transmitting immediately with the harvested energy, i.e., without storing or retrieving energy to/from battery. In essence, the thresholds in the double-threshold policy define an interval within which storing energy is not worth incurring the storage losses. Hence, our work demonstrates how optimal policies need to adapt to the trade-off between scheduling and storage inefficiency. Finally, we formulate the online version of the problem as a dynamic program (Section VII). We observe numerically that the solution of the online dynamic problem formulation also has a double-threshold structure. Due to the complexity of dynamic programming solutions, we propose simpler threshold-based policies and evaluate their performance via simulations (Section VIII) and observe that they perform near-optimal.

\section{System Model}

Time is slotted with unit slot length $\tau=1$ over a finite session of $N$ time slots ${ }^{2}$. The system model is shown in Fig. 1. At the beginning of the $i$ th time slot, the transmitter harvests an energy in the amount of $E_{i} \geq 0$ units. It retrieves an additional $r_{i}$ units of energy from the battery, and allocates $s_{i}$ for storage in the battery. This leaves the energy

$$
p_{i}=E_{i}-s_{i}+r_{i}
$$

for transmission in the $i$ th time slot. The battery has a storing efficiency of $0 \leq \eta \leq 1$ : when $s_{i}$ units of energy is allocated for storing, only $\eta s_{i}$ units can be stored for future use and

\footnotetext{
${ }^{2}$ We consider unit length time slots solely for ease of presentation. The results extend trivially to any positive slot length $\tau>0$.
} 


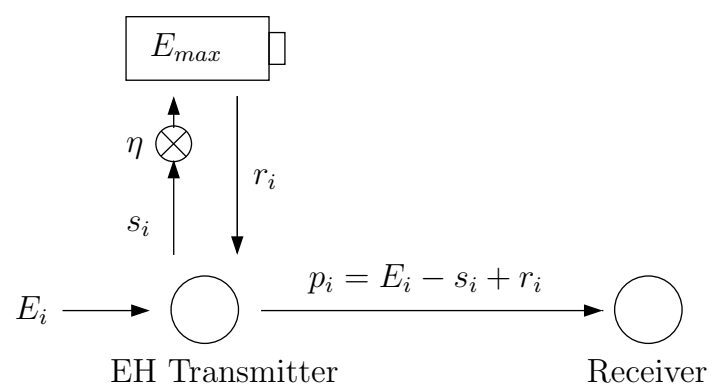

Fig. 1. Energy harvesting transmitter with inefficient storage and finite-sized battery over a Gaussian channel.

$(1-\eta) s_{i}$ units of energy is lost due to storage inefficiency ${ }^{3}$.

The power policy of the node consists of energy values chosen for storage and retrieval, namely $s_{i}$ for storage and $r_{i}$ for retrieval, respectively, at time slots $i=1, \ldots, N$. From these two variables, transmit power $p_{i}$ is calculated using (1) for each time slot of unit duration. Note that by definition, $s_{i} \geq 0, r_{i} \geq 0$ and $p_{i} \geq 0$ for $i=1, \ldots, N$. From (1), the last condition imposes that $E_{i}+r_{i}-s_{i} \geq 0$ on $r_{i}$ and $s_{i}$. Furthermore, the energy drawn from the battery cannot exceed the energy stored in the battery up to any time slot. Following [11], we refer to this constraint as energy causality. Let the initial charge of the battery be $E_{0}$. Denoting the amount of energy in the battery at time slot $i$ as $B_{i}$, the energy causality constraints are given by

$$
B_{i}=E_{0}+\sum_{j=1}^{i}\left(\eta s_{j}-r_{j}\right) \geq 0, \quad i=1, \ldots, N .
$$

In addition, the battery has a maximum storage capacity (size) of $E_{\max }$, and the energy in excess of this capacity is lost if attempted to be stored. Clearly, it is sub-optimal to allow such energy overflows, and it is shown in [12] that restricting power policies to those avoiding overflows yields an optimal policy. Hence, we enforce a set of no-energy-overflow [12] constraints

$$
B_{i}=E_{0}+\sum_{j=1}^{i}\left(\eta s_{j}-r_{j}\right) \leq E_{\max }, \quad i=1, \ldots, N,
$$

which ensure that the energy allocated for storage does not exceed the capacity of the battery at any time slot.

We consider an additive white Gaussian noise communication channel with a fading coefficient of $h_{i}$ at time slot $i$. With allocated transmit power $p$ and fading $h$, the communication rate in a slot is given by

$$
g(p)=\frac{1}{2} \log (1+h p) .
$$

Under this channel model, we consider the problem of maximizing the average throughput of this system, i.e., maximizing the average of $g\left(p_{i}\right)$ over a duration of $N$ time slots, by choosing the optimal power policy $\left\{\left(s_{i}, r_{i}\right)\right\}_{i=1}^{N}$. This requires adapting the power policy to the harvesting process, inefficient storage, and channel coefficients.

\footnotetext{
${ }^{3}$ Similarly, a loss may occur when energy is retrieved from the battery. In this work, these two losses are combined in the model in $\eta$, which effectively represents the fraction of energy that can be drawn from the battery per unit energy stored.
}

\section{Optimal TRANSMission POLICY FOR AN INFINITE-SIZED BATTERY, $E_{\max }=\infty$}

We first consider a non-fading channel, i.e., $h_{i}=h$ for all $i$, and an infinite-sized battery, $E_{\max }=\infty$. The throughput maximization problem for the model in Fig. 1 over an $N$-slot communication session is expressed as

$$
\begin{aligned}
& \max _{\left\{s_{i}, r_{i}\right\}} \sum_{i=1}^{N} g\left(E_{i}-s_{i}+r_{i}\right), \\
& \text { s.t. } \quad E_{0}+\sum_{j=1}^{i}\left(\eta s_{j}-r_{j}\right) \geq 0, \quad i=1, \ldots, N, \\
& E_{i}-s_{i}+r_{i} \geq 0, \quad i=1, \ldots, N, \\
& s_{i} \geq 0, r_{i} \geq 0, \quad i=1, \ldots, N,
\end{aligned}
$$

where $g(p)$ is given in (4). We first present the following lemma which states that it is sub-optimal to store and retrieve energy simultaneously in the same time slot for $\eta<1$. The $\eta=1$ case is omitted since efficient storing and restoring in the same time slot is equivalent to not storing for this case.

Lemma 1 For $\eta<1$, the solution to (5) satisfies $s_{i} r_{i}=0$ for all $i$, i.e., the optimal policy never stores and retrieves energy simultaneously.

Proof: Let $\left\{\left(s_{i}, r_{i}\right)\right\}_{i=1}^{N}$ be a feasible power policy which satisfies $s_{j} r_{j}>0$ for some $j$. Let

$$
\bar{s}_{j}=\left[s_{j}-r_{j} / \eta\right]^{+}, \quad \bar{r}_{j}=\left[r_{j}-\eta s_{j}\right]^{+},
$$

where $[x]^{+}=\max (0, x)$. For all $i \neq j$, let $\bar{s}_{i}=s_{i}$ and $\bar{r}_{i}=r_{i}$. Note that the battery dynamics in (2) are unaffected by this change, since $\eta s_{i}-r_{i}=\eta \bar{s}_{i}-\bar{r}_{i}$, for all $i$. Therefore, the policy $\left\{\left(\bar{s}_{i}, \bar{r}_{i}\right)\right\}_{i=1}^{N}$ is feasible. On the other hand, the resulting transmit power $\bar{p}_{j}$ at time slot $j$ becomes

$$
\bar{p}_{j}=E_{j}-\bar{s}_{j}+\bar{r}_{j}= \begin{cases}E_{j}-s_{j}+r_{j} / \eta, & \text { if } \eta s_{j} \geq r_{j}, \\ E_{j}-\eta s_{j}+r_{j}, & \text { otherwise }\end{cases}
$$

and consequently $\bar{p}_{j}>p_{j}$ due to $\eta<1, s_{j}>0$ and $r_{j}>$ 0 . Since the rate $g(p)$ is increasing in $p$, we have $g\left(\bar{p}_{j}\right)>$ $g\left(p_{j}\right)$, while $g\left(\bar{p}_{i}\right)=g\left(p_{i}\right)$ for $i \neq j$. Hence, the power policy $\left\{\left(\bar{s}_{i}, \bar{r}_{i}\right)\right\}_{i=1}^{N}$ achieves a larger throughput than $\left\{\left(s_{i}, r_{i}\right)\right\}_{i=1}^{N}$, and the latter policy cannot be optimal.

Lemma 1 shows that we can restrict our search for the optimal policy to those that do not store and retrieve energy simultaneously at any time. We remark that simultaneously charging and discharging a battery may or may not be physically possible, but through Lemma 1, we show that it is mathematically sub-optimal for our problem.

\section{A. Double-Threshold Policy}

We next observe a property of the optimal power policy. Since all constraints are linear and $g(p)$ is concave in $p$, the problem in (1) is a convex optimization problem. Hence, 
Karush-Kuhn-Tucker (KKT) conditions are necessary and sufficient for optimality. The Lagrangian of (5) is

$$
\begin{gathered}
\mathcal{L}=\sum_{i=1}^{N}\left(g\left(E_{i}-s_{i}+r_{i}\right)+\lambda_{i}\left(E_{0}+\sum_{j=1}^{i}\left(\eta s_{j}-r_{j}\right)\right)\right. \\
\left.+\mu_{i}\left(E_{i}-s_{i}+r_{i}\right)+\sigma_{i} s_{i}+\nu_{i} r_{i}\right)
\end{gathered}
$$

where $\lambda_{i}, \mu_{i}, \sigma_{i}$ and $\nu_{i}, i=1, \ldots, N$ are non-negative Lagrange multipliers corresponding to the energy causality, non-negativity of power, and non-negativity of stored and retrieved energy, respectively. The KKT optimality conditions are found by taking the derivatives with respect to $s_{i}$ and $r_{i}$ for $i=1, \ldots, N$ as

$$
\begin{aligned}
-\frac{h}{1+h p_{i}}+\eta \sum_{j=i}^{N} \lambda_{j}-\mu_{i}+\sigma_{i} & =0, \quad i=1, \ldots, N, \\
\frac{h}{1+h p_{i}}-\sum_{j=i}^{N} \lambda_{j}+\mu_{i}+\nu_{i} & =0, \quad i=1, \ldots, N,
\end{aligned}
$$

with the complementary slackness conditions

$$
\begin{array}{rlrl}
\lambda_{i}\left(E_{0}+\sum_{j=1}^{i}\left(\eta s_{j}-r_{j}\right)\right) & =0, & & i=1, \ldots, N, \\
\mu_{i}\left(E_{i}-s_{i}+r_{i}\right) & =0, & i=1, \ldots, N, \\
\sigma_{i} s_{i}=0, \quad \nu_{i} r_{i} & =0, & i=1, \ldots, N .
\end{array}
$$

From (9) and (10), we find the optimal transmit powers $p_{i}$ as

$$
\begin{aligned}
p_{i} & =\frac{1}{\eta \sum_{j=i}^{N} \lambda_{j}-\mu_{i}+\sigma_{i}}-\frac{1}{h} \\
& =\frac{1}{\sum_{j=i}^{N} \lambda_{j}-\mu_{i}-\nu_{i}}-\frac{1}{h}, \quad i=1, \ldots, N .
\end{aligned}
$$

We define two sets of thresholds, $p_{s i}$ and $p_{r i}$, as

$$
\begin{aligned}
& p_{s i}=\frac{1}{\eta \sum_{j=i}^{N} \lambda_{j}}-\frac{1}{h}, \quad i=1, \ldots, N, \\
& p_{r i}=\frac{1}{\sum_{j=i}^{N} \lambda_{j}}-\frac{1}{h}, \quad i=1, \ldots, N .
\end{aligned}
$$

Note that these variables satisfy

$$
p_{s i} \geq p_{r i}, \quad i=1, \ldots, N
$$

and are related as

$$
\frac{1+h p_{r i}}{1+h p_{s i}}=\eta, \quad i=1, \ldots, N
$$

We note that whenever $p_{i}>0$, we have $\mu_{i}=0$ from (11b). Then, from the first equality in (12), since $\sigma_{i} \geq 0$, we have $p_{i} \leq p_{s i}$. Similarly, from the second equality in (12), since $\nu_{i} \geq 0$, we have $p_{i} \geq p_{r i}$. Therefore, for $p_{i}>0$, we have

$$
p_{s i} \geq p_{i} \geq p_{r i} .
$$

We refer to $p_{s i}$ and $p_{r i}$ as thresholds: when transmit power $p_{i}>0$, it must be larger than the lower threshold $p_{r i}$, and smaller than the upper threshold $p_{s i}$. In the following lemma, we show that charging and discharging are also related to these thresholds in the optimal policy.

Lemma 2 Whenever the battery is being charged, i.e., $s_{i}>0$, a non-zero transmit power must satisfy $p_{i}=p_{s i}$. Conversely, whenever the battery is being discharged, i.e., $r_{i}>0$, a nonzero transmit power must satisfy $p_{i}=p_{\text {ri }}$.

Proof: For a non-zero transmit power $p_{i}>0$, due to (11b) we have $\mu_{i}=0$. When the battery is being charged, i.e., $s_{i}>0$, from (11c), we get $\sigma_{i}=0$. Substituting this in the first equality in (12) yields $p_{i}=p_{s i}$. When the battery is being discharged, i.e., $r_{i}>0$, from (11c), we get $\nu_{i}=0$. Substituting this in the second equality in (12) yields $p_{i}=p_{r i}$.

Due to Lemma 2, we call $p_{s i}$ the storing threshold and $p_{r i}$ the retrieving threshold. We observe from Lemma 1 that we have either $s_{i}>0$ and $r_{i}=0$, or $s_{i}=0$ and $r_{i}>0$, or $s_{i}=0$ and $r_{i}=0$. When $s_{i}=r_{i}=0$, from (1), we have $p_{i}=E_{i}$, which must satisfy (16). These conditions show that there is a double-threshold policy on $p_{i}$. Specifically, when the battery is being charged, the transmit power equals the storing threshold $p_{s i}$; and when the battery is being discharged, the transmit power equals the retrieving threshold $p_{r i}$. If the battery is neither being charged or discharged, i.e., the battery is passive, then $p_{i}=E_{i}$, i.e., the transmitter uses all the harvested energy in the current slot.

Theorem 1 The power policy solving (5) has the following double-threshold structure:

a) If $E_{i}>p_{s i}$, then $p_{i}=\left[p_{s i}\right]^{+}$. Consequently, $s_{i}=E_{i}-$ $\left[p_{s i}\right]^{+}>0$ and $r_{i}=0$ (storing).

b) If $E_{i}<p_{r i}$, then $p_{i}=p_{r i}$. Consequently, $s_{i}=0$ and $r_{i}=p_{r i}-E_{i}>0$ (retrieving).

c) If $p_{s i} \geq E_{i} \geq p_{r i}$, then $s_{i}=r_{i}=0$ and $p_{i}=E_{i}$ (passive).

Proof: We prove each case separately:

a) Consider the case $E_{i}>p_{s i}$. From (1) and Lemma 1, we have $s_{i} \leq E_{i}$. We consider the three distinct cases $s_{i}=0$, $0<s_{i}<E_{i}$ and $s_{i}=E_{i}$ as follows. When $s_{i}=0$, from (1), we get $p_{i} \geq E_{i}$. Together with $E_{i}>p_{s i}$, this contradicts (16). Therefore, this case cannot be optimal. When $E_{i}>s_{i}>0$, from (1) we get $p_{i}>0$ and from Lemma 2 we have $p_{i}=p_{s i}$. Finally, when $s_{i}=E_{i}$ and therefore $r_{i}=0$, (1) yields $p_{i}=0$. Since $\sigma_{i}=0$ from (11c), substituting in (12) gives $p_{s i} \leq p_{i}=$ 0 . Hence, for all possible sub-cases in this case, $p_{i}=\left[p_{s i}\right]^{+}$.

b) Consider the case $E_{i}<p_{r i}$. We consider the three distinct cases, $r_{i}=0$ and $p_{i}>0, r_{i}=0$ and $p_{i}=0$, and $r_{i}>0$ as follows. When $r_{i}=0$ and $p_{i}>0$, from (1), we get $p_{i} \leq E_{i}$. Together with $E_{i}<p_{r i}$, this contradicts (16), and therefore this case cannot be optimal. When $r_{i}=0$ and $p_{i}=0$, from (1) we have $s_{i}=E_{i}$, implying that $\sigma_{i}=0$ due to (11c). From (12) and (14), we get $p_{r i} \leq p_{i}=0$, which contradicts $E_{i}<p_{r i}$. Therefore, this case cannot be optimal. Finally, when $r_{i}>0$, this implies $p_{i}>0$ due to (1) and Lemma 1. From Lemma 2 we have $p_{i}=p_{r i}$. Hence, for the $E_{i}<p_{r i}$ case, the only possible transmit power is $p_{i}=p_{r i}$. 
c) Consider the case $p_{s i} \geq E_{i} \geq p_{r i}$. When $s_{i}>0$, then from (1) and Lemma 1, we get $p_{i}<E_{i}$. Due to $p_{s i} \geq E_{i}$, this contradicts Lemma 2. Therefore, this case cannot be optimal. On the other hand, when $r_{i}>0$, then (1) and Lemma 1 yield $p_{i}>E_{i}$. Due to $E_{i} \geq p_{r i}$, this contradicts Lemma 2, and therefore this case cannot be optimal. Hence, $s_{i}=r_{i}=0$ in this case, yielding $p_{i}=E_{i}$ from (1).

In summary, Theorem 1 shows that the optimal policy is a double-threshold policy that can be expressed as

$$
\begin{aligned}
p_{i} & =\min \left(\max \left(E_{i}, p_{r i}\right),\left[p_{s i}\right]^{+}\right), \\
s_{i} & =\left[E_{i}-p_{i}\right]^{+}, \quad r_{i}=\left[p_{i}-E_{i}\right]^{+} .
\end{aligned}
$$

To find the entire policy, it remains to find the thresholds $p_{s i}$ and $p_{r i}$ for $i=1, \ldots, N$, which we describe next.

\section{B. Finding the Thresholds}

To determine the thresholds defined in (13), we make the observations stated in the following two lemmas: Lemma 3 states that these thresholds are non-decreasing in general, and remain constant in stretches of time slots when there is energy in the battery. Therefore, they only potentially increase when the battery is depleted. Lemma 4 states that the battery must be depleted by the end of the communication session, otherwise the throughput can be increased by retrieving and using the remaining energy in the battery in the last slot.

Lemma 3 The thresholds $p_{s i}$ and $p_{r i}$ in (13) are nondecreasing, and remain constant unless the battery is depleted, i.e., $p_{s(i+1)}=p_{s i}$ and $p_{r(i+1)}=p_{r i}$ for all $i$ when $B_{i}>0$.

Proof: The non-decreasing property follows from $\lambda_{i} \geq 0$ in (13a)-(13b). The second property in the lemma is a consequence of the complementary slackness condition in (11a), which implies that when $B_{i}>0$ we have $\lambda_{i}=0$ and $p_{s i}$ and $p_{\text {ri }}$ remain constant from (13a)-(13b).

Lemma 4 In the optimal policy, the battery is depleted at the end of the session, i.e., $B_{N}=0$.

Proof: The proof is by contradiction. Let $\left\{\left(s_{i}, r_{i}\right)\right\}_{i=1}^{N}$ be a feasible policy with $B_{N}>0$. Let $\bar{s}_{i}=s_{i}$ for $i=1, \ldots, N$, $\bar{r}_{i}=r_{i}$ for $i=1, \ldots, N-1$, and $\bar{r}_{N}=r_{N}+B_{N}$. Note that $\left\{\left(\bar{s}_{i}, \bar{r}_{i}\right)\right\}_{i=1}^{N}$ is a feasible policy. For this new policy, we have $g\left(\bar{p}_{i}\right)=g\left(p_{i}\right)$ for $i=1, \ldots, N-1$ and $g\left(\bar{p}_{N}\right)>g\left(p_{N}\right)$, yielding a larger throughput. Hence, $\left\{\left(s_{i}, r_{i}\right)\right\}_{i=1}^{N}$ cannot be optimal.

In light of Lemmas 3 and 4, we seek a set of thresholds that are non-decreasing for all $i$, only increasing when $B_{i}=0$, and depleting the battery at the end of the transmission. Note that it suffices to find $p_{s i}$ values only, and $p_{r i}$ can be calculated from the fixed relationship in (15). For this purpose, we propose the algorithm below.

Algorithm 1 Start from time slot $j=1$. Using linear search, find the largest threshold $p_{s} \geq 0$, and the corresponding $p_{r}$ from (15), for which the transmit power policy given by (17) is feasible in $i=j, \ldots, N$. Find the smallest $\ell>j$ such that $B_{\ell}=0$, and assign optimal thresholds $p_{s}$ and $p_{r}$ to time slots $i=j, \ldots, \ell$. If $\ell<N$, repeat the above procedure starting from $j=\ell+1$.

The procedure in Algorithm 1 ensures that the resulting thresholds are non-decreasing and remain constant while the battery is not empty, as required by Lemma 3 . The nondecreasing property can be seen as follows: At a step starting from time slot $j$, the previous threshold $p_{s(j-1)}$ is feasible in $i=j, \ldots, N$ by construction. Hence, the new threshold $p_{s j} \geq p_{s(j-1)}$. Next, we prove the optimality of these thresholds.

Theorem 2 The policy in (17) with thresholds $\left\{\left(p_{s i}^{*}, p_{r i}^{*}\right)\right\}_{i=1}^{N}$ found using Algorithm 1 is the solution to (5).

Proof: We show that using $p_{s i}^{*}$ and $p_{r i}^{*}, i=1, \ldots, N$, a set of Lagrange multipliers satisfying all KKT conditions in (9)-(11) can be found. Note that $p_{s i}^{*}$ and $p_{r i}^{*}$ are non-decreasing. Let

$$
\lambda_{i}=\frac{1}{\eta\left(p_{s i}^{*}+1 / h\right)}-\frac{1}{\eta\left(p_{s(i+1)}^{*}+1 / h\right)}
$$

for $i=1, \ldots, N$, with $p_{s(N+1)}^{*}=\infty$ by definition. This satisfies $\lambda_{i} \geq 0$ since $p_{s i}^{*}$ is non-decreasing, and satisfies (11a) since $p_{s i}^{*}$ only changes when $B_{i}=0$.

Next, let $\mu_{i}=0, i=1, \ldots, N$, which satisfy (11b). Since $p_{s i}^{*} \geq 0$ and $p_{r i}^{*} \geq 0$ by construction, from (17a) we get $p_{s i}^{*} \geq p_{i}^{*} \geq p_{r i}^{*}$ for all $i$. Calculate $\sigma_{i}$ and $\nu_{i}$ from (12) as

$$
\sigma_{i}=\frac{h}{1+h p_{i}^{*}}-\frac{h}{1+h p_{s i}^{*}}, \quad \nu_{i}=\frac{h}{1+h p_{r i}^{*}}-\frac{h}{1+h p_{i}^{*}} .
$$

Note that these values are non-negative since $p_{s i}^{*} \geq p_{i}^{*} \geq p_{r i}^{*}$. Furthermore, they satisfy (11c) as follows: when $s_{i}>0,(17 \mathrm{~b})$ implies $E_{i}>p_{i}^{*}$. Thus, from (17a), we have $p_{i}^{*}=\left[p_{s i}^{*}\right]^{+}=$ $p_{s i}^{*}$, and therefore (19) yields $\sigma_{i}=0$. Similarly, when $r_{i}>0$, (17b) implies $E_{i}<p_{i}^{*}$, and therefore (17a) implies $p_{i}^{*}=p_{r i}^{*}$. Hence, (19) yields $\nu_{i}=0$.

An example run of Algorithm 1 and the resulting optimal transmission policy is shown in Fig. 2. The example is over $N=5$ time slots with storage efficiency $\eta=0.5$, energy harvests $\left\{E_{i}\right\}=\{9,4,2,13,4\}$, initial charge $E_{0}=0$, and $h=1$. Starting from $j=1$, the largest feasible thresholds satisfying (15) are found as $p_{s}=7$ and $p_{r}=3$, depleting the battery at the end of time slot $i=3$. Setting these thresholds for $i=1,2,3$, the second set of thresholds starting from $j=4$ are found as $p_{s}=11$ and $p_{r}=5$, depleting the battery at the end of time slot $i=5=N$. With these thresholds, the optimal transmit powers $p_{i}^{*}$ are shown in red. Energy stored at the first time slot, marked as I, is retrieved at the third time slot, marked as II. Similarly, energy stored in the fourth time slot marked as III is retrieved and consumed entirely in the fifth time slot marked as IV. Note that since $p_{s i}^{*} \geq E_{i} \geq p_{r i}^{*}$ at $i=2$, no charging or discharging takes place. In this slot, transmitter is in the passive state in Theorem 1, and uses only the incoming energy for transmission, i.e., $p_{i}^{*}=E_{i}$. 


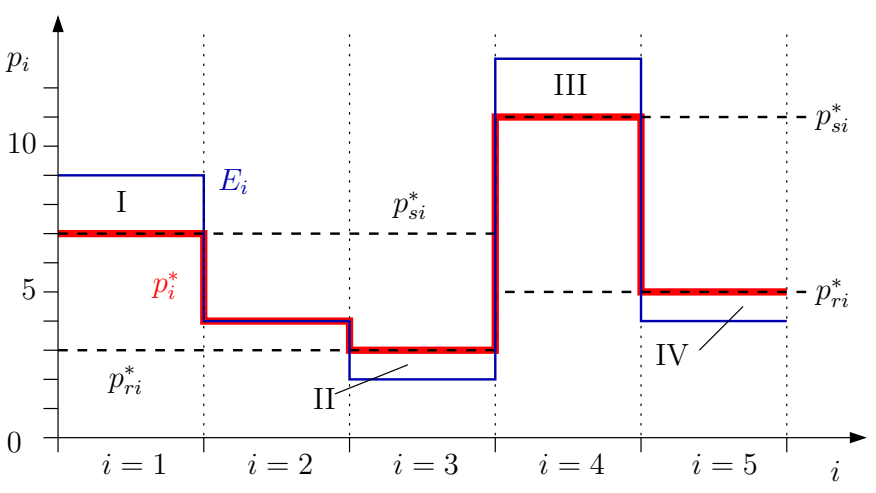

Fig. 2. Example optimal policy with transmission power thresholds $p_{s i}$ and $p_{\text {ri }}$.

The optimal policy derived in this section can be shown to converge to the results of [11] when energy storage is ideal. This is when $\eta=1$, and (15) yields $p_{s i}=p_{\text {ri }}$ for all $i$. Due to (17a), this implies $p_{i}=\left[p_{s i}\right]^{+}$at all times. Consequently, the optimal policy consists of piecewise constant power transmissions, with the transmit power increasing only at instances of empty battery due to Lemma 3. This coincides with the result with ideal battery in [11] which finds the longest constant power stretches, and changes the power only when the battery is depleted. As battery efficiency $\eta$ decreases, the two thresholds $p_{s i}$ and $p_{r i}$ separate, yielding a larger region for the passive state. Our optimal policy describes the transition from the constant power policy in one extreme, $\eta=1$, to the spend what you get policy without storage in the other, $\eta=0$.

\section{Optimal TRANSMisSiON POLICY FOR A FINITE-SIZED BATTERY, $E_{\max }<\infty$}

In practice, energy storage devices are of finite size. In this section, we extend the infinite-sized battery problem in (5) to the case of a finite-sized battery by including the additional noenergy-overflow constraint in (3). For a battery of size $E_{\max }$, the throughput maximization problem becomes

$$
\begin{aligned}
& \max _{\left\{s_{i}, r_{i}\right\}} \sum_{i=1}^{N} g\left(E_{i}-s_{i}+r_{i}\right) \\
& \text { s.t. } \quad 0 \leq E_{0}+\sum_{j=1}^{i}\left(\eta s_{j}-r_{j}\right) \leq E_{\max }, \quad i=1, \ldots, N \\
& E_{i}-s_{i}+r_{i} \geq 0, \quad i=1, \ldots, N \\
& s_{i} \geq 0, r_{i} \geq 0, \quad i=1, \ldots, N
\end{aligned}
$$

where $g(p)$ is defined in (4). The Lagrangian of (20) is

$$
\begin{gathered}
\mathcal{L}=\sum_{i=1}^{N}\left(g\left(E_{i}-s_{i}+r_{i}\right)+\lambda_{i}\left(E_{0}+\sum_{j=1}^{i}\left(\eta s_{j}-r_{j}\right)\right)\right. \\
-\beta_{i}\left(E_{0}+\sum_{j=1}^{i}\left(\eta s_{j}-r_{j}\right)-E_{\max }\right) \\
\left.+\mu_{i}\left(E_{i}-s_{i}+r_{i}\right)+\sigma_{i} s_{i}+\nu_{i} r_{i}\right)
\end{gathered}
$$

where $\beta_{i}, i=1, \ldots, N$ are the non-negative Lagrange multipliers for the no-energy-overflow constraints. The KKT optimality conditions are

$$
\begin{array}{r}
-\frac{h}{1+h p_{i}}+\eta \sum_{j=i}^{N}\left(\lambda_{j}-\beta_{j}\right)-\mu_{i}+\sigma_{i}=0, \quad i=1, \ldots, N, \\
\frac{h}{1+h p_{i}}-\sum_{j=i}^{N}\left(\lambda_{j}-\beta_{j}\right)+\mu_{i}+\nu_{i}=0, \quad i=1, \ldots, N .
\end{array}
$$

The complementary slackness conditions corresponding to $\beta_{i}$ are

$$
\beta_{i}\left(E_{0}+\sum_{j=1}^{i}\left(\eta s_{j}-r_{j}\right)-E_{\max }\right)=0, \quad i=1, \ldots, N
$$

which, together with those listed in (11), constitute the complementary slackness conditions for the problem in (20). From (22) and (23), we find the optimal transmit powers $p_{i}$ as

$$
\begin{aligned}
p_{i} & =\frac{1}{\eta \sum_{j=i}^{N}\left(\lambda_{j}-\beta_{j}\right)-\mu_{i}+\sigma_{i}}-\frac{1}{h} \\
& =\frac{1}{\sum_{j=i}^{N}\left(\lambda_{j}-\beta_{j}\right)-\mu_{i}-\nu_{i}}-\frac{1}{h}, \quad i=1, \ldots, N .
\end{aligned}
$$

In view of the new multipliers $\beta_{i}$, we update the definition of thresholds, $p_{s i}$ and $p_{r i}$, as

$$
\begin{aligned}
& p_{s i}=\frac{1}{\eta \sum_{j=i}^{N}\left(\lambda_{j}-\beta_{j}\right)}-\frac{1}{h}, \quad i=1, \ldots, N, \\
& p_{r i}=\frac{1}{\sum_{j=i}^{N}\left(\lambda_{j}-\beta_{j}\right)}-\frac{1}{h}, \quad i=1, \ldots, N,
\end{aligned}
$$

which satisfy (14) and (15). Observing that $\mu_{i}=0$ when $p_{i}>0$, (16) must also hold for the optimal policy.

\section{A. Finding the Thresholds for a Finite-Sized Battery}

In this finite-sized battery case, Lemmas 1, 2 and 4 continue to hold, i.e., in this finite-sized battery case also $s_{i} r_{i}=0$ (simultaneous storing and retrieval is sub-optimal), if $s_{i}>0$ then $p_{i}=p_{s i}$ (when storing, the power must be equal to the storing threshold), if $r_{i}>0$ then $p_{i}=p_{r i}$ (when retrieving, the power must be equal to the retrieving threshold), and $B_{N}=0$ (the battery must be depleted at the end of the communication session). However, due to $\beta_{i}$, the new thresholds in (26) no longer satisfy Lemma 3, i.e., the new thresholds are no longer monotone. Instead, they satisfy the property stated in the following lemma.

Lemma 5 The thresholds $p_{s i}$ and $p_{r i}$ in (26) are nondecreasing while $B_{i}<E_{\max }$, and non-increasing while $B_{i}>0$. Consequently, they remain constant if the battery is not depleted or full, i.e., $p_{s(i+1)}=p_{s i}$ and $p_{r(i+1)}=p_{r i}$ for all $i$ while $0<B_{i}<E_{\max }$.

Proof: For $B_{i}<E_{\max }$, (24) gives $\beta_{i}=0$. Substituting in (26), this implies that $p_{s i}$ and $p_{r i}$ are non-decreasing. 
Similarly, for $B_{i}>0$, (11a) gives $\lambda_{i}=0$. Substituting in (26), this implies that $p_{s i}$ and $p_{r i}$ are non-increasing. Finally, for $0<B_{i}<E_{\max }$, (11a) and (24) give $\lambda_{i}=0$ and $\beta_{i}=0$, which yields $p_{s(i+1)}=p_{s i}$ and $p_{r(i+1)}=p_{r i}$ from (26), which implies that $p_{s i}$ and $p_{r i}$ remain constant.

Hence, we are looking for a feasible set of thresholds satisfying Lemmas 1, 2, 4 and 5. We propose the following algorithm to find the optimal policy.

Algorithm 2 Start from time slot $j=1$. Find the largest threshold $p_{s} \geq 0$, and the corresponding $p_{r}$ from (15), such that the transmit power policy given by (17) does not violate (2) first, i.e., either the policy is feasible for $i=j, \ldots, N$, or (3) is violated before (2). Find the smallest $\ell>j$ such that $B_{\ell}=0$ or $B_{\ell}=E_{\max }$, and assign optimal thresholds $p_{s}$ and $p_{r}$ to the time slots $i=j, \ldots, \ell$. If $\ell<N$, repeat the above procedure starting from $j=\ell+1$.

The next lemma shows that the thresholds found by this algorithm satisfy Lemma 5.

Lemma 6 The thresholds found by Algorithm 2 satisfy the conditions in Lemma 5.

Proof: Starting from some $j$, let the algorithm output $p_{s j}, p_{r j}$ and $\ell$. Consider the case $B_{\ell}=0$. Then, the constant threshold $p_{s j}$ must yield a full battery at some $k>\ell$, or be feasible until $i=N$, since otherwise a smaller $p_{s j}$ would have been chosen by the algorithm. Hence, starting from time slot $\ell$, the next threshold cannot be less than $p_{s j}$. Now, consider the case $B_{\ell}=E_{\max }$. Then, the constant threshold $p_{s j}$ violates (2) or depletes the battery at some $k>\ell$ by construction. Hence, starting from time slot $\ell$, the next threshold cannot be greater than $p_{s j}$.

As a result of Lemma 6, we have that the thresholds found by Algorithm 2 are non-decreasing if $B_{i}=0$, non-increasing if $B_{i}=E_{\max }$, and by construction constant in between. Next, we prove the optimality of the resulting policy.

Theorem 3 The policy in (17) with thresholds $\left\{\left(p_{s i}^{*}, p_{r i}^{*}\right)\right\}_{i=1}^{N}$ found using Algorithm 2 is the solution to (20).

Proof: We show that using $p_{s i}^{*}$ and $p_{r i}^{*}, i=1, \ldots, N$, a set of Lagrange multipliers satisfying all KKT conditions in (11) and (22)-(24) can be found. First, note that $p_{s i}^{*}$ and $p_{r i}^{*}$ are constant unless the battery is depleted or full, non-decreasing if $B_{i}=0$ and non-increasing if $B_{i}=E_{\max }$, as shown in Lemma 6. Let

$$
\begin{aligned}
& \lambda_{i}=\left[\frac{1}{\eta\left(p_{s i}^{*}+1 / h\right)}-\frac{1}{\eta\left(p_{s(i+1)}^{*}+1 / h\right)}\right]^{+}, \\
& \beta_{i}=\left[\frac{1}{\eta\left(p_{s(i+1)}^{*}+1 / h\right)}-\frac{1}{\eta\left(p_{s i}^{*}+1 / h\right)}\right]^{+},
\end{aligned}
$$

for $i=1, \ldots, N$, with $p_{s(N+1)}^{*}=\infty$ by definition. These satisfy (11a) and (24) due to Lemma 6. The rest of the
Lagrangian multipliers are found as in the proof of Theorem 2, by replacing (12) with (25).

The policy for the finite battery case in this section converges to the previous results for the ideal battery case studied in [12], when $\eta=1$. In this case, the thresholds are equal and thus the optimal policy is a constant power policy as in [12]. For equal thresholds, the conditions in Lemma 5 coincide with those in [12, Theorem 1].

\section{Optimal Transmission POLICY FOR A FADING CHANNEL}

We now consider a fading channel, where the fading channel coefficient $h_{i}, i=1, \ldots, N$, is constant throughout time slot $i$, but changes from one time slot to another. The coefficients are known non-causally at the transmitter. This is an extension of [13] to the inefficient energy storage case; see also [14]. The instantaneous rate in slot $i$ is given in (4), which we will denote as $g(p, h)$ in this section, to emphasize its dependence on the channel gain $h$.

The throughput maximization problem in a fading channel for a transmitter with a finite-sized battery becomes

$$
\begin{aligned}
& \max _{\left\{s_{i}, r_{i}\right\}} \sum_{i=1}^{N} g\left(E_{i}-s_{i}+r_{i}, h_{i}\right) \\
& \text { s.t. } \quad 0 \leq E_{0}+\sum_{j=1}^{i}\left(\eta s_{j}-r_{j}\right) \leq E_{\max }, \quad i=1, \ldots, N \\
& E_{i}-s_{i}+r_{i} \geq 0, \quad i=1, \ldots, N \\
& s_{i} \geq 0, \quad r_{i} \geq 0, \quad i=1, \ldots, N
\end{aligned}
$$

yielding the KKT optimality conditions

$$
\begin{array}{r}
-\frac{h_{i}}{1+h_{i} p_{i}}+\eta \sum_{j=i}^{N}\left(\lambda_{j}-\beta_{j}\right)-\mu_{i}+\sigma_{i}=0, \quad i=1, \ldots, N, \\
\frac{h_{i}}{1+h_{i} p_{i}}-\sum_{j=i}^{N}\left(\lambda_{j}-\beta_{j}\right)+\mu_{i}+\nu_{i}=0, \quad i=1, \ldots, N,
\end{array}
$$

and the complementary slackness conditions in (11) and (24). We note that Lemma 1 holds for the fading case as well, since it only depends on the rate function $g(p, h)$ being nondecreasing in $p$.

For the fading case, we define the following water-level thresholds,

$$
v_{s i}=\frac{1}{\eta \sum_{j=i}^{N}\left(\lambda_{j}-\beta_{j}\right)}, \quad v_{r i}=\frac{1}{\sum_{j=i}^{N}\left(\lambda_{j}-\beta_{j}\right)},
$$

which satisfy

$$
v_{r i}=\eta v_{s i}, \quad i=1, \ldots, N .
$$

With these definitions, we observe that for a positive transmit power, $p_{i}>0,(11 \mathrm{~b})$ gives $\mu_{i}=0$. Therefore, if the battery is being charged, i.e., $s_{i}>0$, from (11c) and (29) we have $p_{i}=v_{s i}-1 / h_{i}$. Similarly, if the battery is being discharged, 

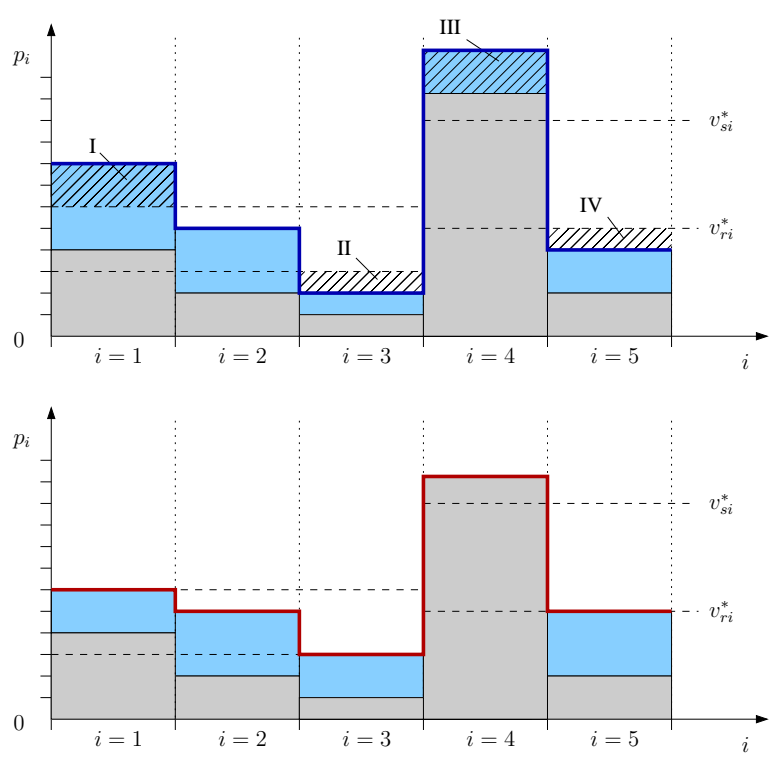

Fig. 3. Directional water-filling with energy storage and retrieval thresholds: (a) The initial water levels with $p_{i}=E_{i}$, and resulting thresholds, and (b) resulting water levels and optimal transmit powers.

i.e., $r_{i}>0,(11 \mathrm{c})$ and (30) yields $p_{i}=v_{r i}-1 / h_{i}$. If $p_{i}=0$, then $s_{i}=E_{i}$ is being stored, and hence $\sigma_{i}=0$. Since $\mu_{i} \geq 0$ by definition, (29) gives $v_{s i}-1 / h_{i}<0$. Hence, for the storing and retrieving cases, the optimal transmit powers are

$p_{i}=\left[v_{s i}-\frac{1}{h_{i}}\right]^{+}$(storing), $\quad p_{i}=\left[v_{r i}-\frac{1}{h_{i}}\right]^{+}$(retrieving).

Note that the thresholds $v_{s i}$ and $v_{r i}$ no longer equal transmit powers directly in these cases, as in Sections III and IV, but set the water-levels over which water-filling is to be performed. In particular, if we can find water-levels $v_{s i}$ and $v_{r i}$ satisfying (32) such that the power policy

$$
p_{i}=\min \left(\max \left(E_{i}, v_{r i}-\frac{1}{h_{i}}\right),\left[v_{s i}-\frac{1}{h_{i}}\right]^{+}\right)
$$

is feasible, and all KKT conditions are satisfied, then this policy is optimal. Finding these water-level thresholds is possible exactly as in Algorithm 2 after replacing the power policy in (17) with (34) and the thresholds $p_{s i}$ and $p_{r i}$ with $v_{s i}$ and $v_{r i}$, respectively.

An example of directional water-filling with thresholds is given in Fig. 3 for a storage efficiency of $\eta=0.5$ and $N=5$. Fading levels and the harvested energy for $i=1, \ldots, 5$ are shown in Fig. 3(a) in gray and blue, respectively. In particular, the height of the grey area represents $h_{i}^{-1}$, and the height of the blue area represents $p_{i}$ in each time slot. The battery capacity is sufficiently large to store all harvested energy in this example. Two pairs of thresholds satisfying (32) are found such that the battery is empty at the end of time slots 3 and 5. Consequently, the thresholds only change at the end of the third time slot. Energy in the areas marked as I and III are stored, and later retrieved and consumed in the areas marked as II and IV, respectively.

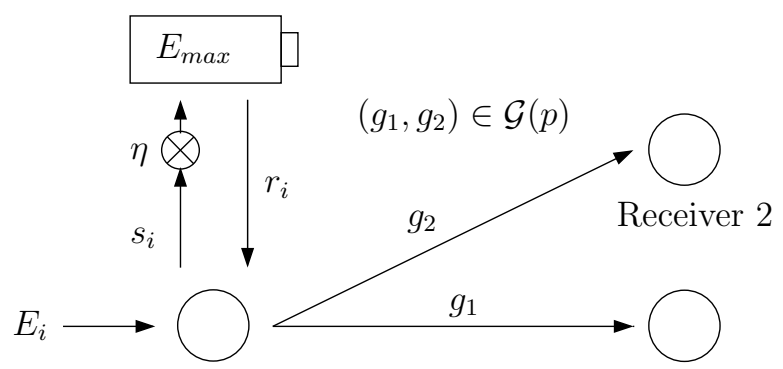

EH Transmitter

Fig. 4. Energy harvesting transmitter with inefficient storage in a Gaussian broadcast channel.

\section{Optimal Transmission Policy for a BROAdCAST CHANNEL}

We next consider a Gaussian broadcast channel, which consists of an energy harvesting transmitter with an inefficient battery, and two receivers, as shown in Fig. 4. At time slot $i$, the transmitter allocates the power $p_{i}$ for transmission, achieving a rate pair $\left(g_{1 i}, g_{2 i}\right) \in \mathcal{G}\left(p_{i}\right)$ where $\mathcal{G}(p)$ is the capacity region for transmit power $p$, given by

$$
\begin{aligned}
\mathcal{G}(p)= & \left\{\left(g_{1}, g_{2}\right) \mid g_{1} \leq \frac{1}{2} \log \left(1+\frac{\alpha p}{\sigma_{1}^{2}}\right),\right. \\
& \left.g_{2} \leq \frac{1}{2} \log \left(1+\frac{(1-\alpha) p}{\alpha p+\sigma_{2}^{2}}\right), 0 \leq \alpha \leq 1\right\},
\end{aligned}
$$

with noise variances $\sigma_{1}^{2}$ and $\sigma_{2}^{2} \geq \sigma_{1}^{2}$ for receivers 1 and 2, respectively [43]. The following lemma presents a property that is common to all capacity regions, in particular for the region in (35), which is an immediate result of time-sharing.

Lemma 7 Let $\left(g_{1}, g_{2}\right) \in \mathcal{G}(p)$ and $\left(\bar{g}_{1}, \bar{g}_{2}\right) \in \mathcal{G}(\bar{p})$. Then,

$$
\left(\lambda g_{1}+(1-\lambda) \bar{g}_{1}, \lambda g_{2}+(1-\lambda) \bar{g}_{2}\right) \in \mathcal{G}(\lambda p+(1-\lambda) \bar{p}) .
$$

For this channel, we characterize the maximum throughput region $\mathcal{G}_{E H}$ as the set of achievable throughput pairs under the energy harvesting constraints in (2) and (3). This is the extension of the maximum departure region in [17, Defn. 1] to the case of inefficient storage. Specifically, we write

$$
\begin{gathered}
\mathcal{G}_{E H}=\left\{\left(\sum_{i=1}^{N} g_{1 i}, \sum_{i=1}^{N} g_{2 i}\right) \mid\left(g_{1 i}, g_{2 i}\right) \in \mathcal{G}\left(E_{i}-s_{i}+r_{i}\right),\right. \\
\left.s_{i}, r_{i} \geq 0, E_{i}-s_{i}+r_{i} \geq 0,(2), \text { (3) }\right\} .
\end{gathered}
$$

We first present the following result, which is an extension of [15, Lemma 2] to the case of inefficient energy storage.

\section{Lemma 8 The throughput region $\mathcal{G}_{E H}$ is convex.}

Proof: Let $\left\{\left(s_{i}, r_{i}\right)\right\}_{1}^{N}$ and $\left\{\left(s_{i}^{\prime}, r_{i}^{\prime}\right)\right\}_{1}^{N}$, be two feasible policies yielding transmit powers $\left\{p_{i}\right\}_{1}^{N}$ and $\left\{p_{i}^{\prime}\right\}_{1}^{N}$, and achieving rate pairs $\left\{\left(g_{1 i}, g_{2 i}\right)\right\}_{1}^{N}$ and $\left\{\left(g_{1 i}^{\prime}, g_{2 i}^{\prime}\right)\right\}_{1}^{N}$, respectively. Let $\bar{s}_{i}=\lambda s_{i}+(1-\lambda) s_{i}^{\prime}$ and $\bar{r}_{i}=\lambda r_{i}+(1-\lambda) r_{i}^{\prime}, i=1, \ldots, N$, 
which yields $\bar{p}_{i}=\lambda p_{i}+(1-\lambda) p_{i}^{\prime}, i=1, \ldots, N$. Then, due to Lemma 7, $\left\{\left(\bar{s}_{i}, \bar{r}_{i}\right)\right\}_{1}^{N}$ can achieve the rates $\bar{g}_{j i} \geq$ $\lambda g_{j i}+(1-\lambda) g_{j i}^{\prime}, j=1,2$. Furthermore, the policy $\left\{\left(\bar{s}_{i}, \bar{r}_{i}\right)\right\}_{1}^{N}$ is feasible since (2) and (3) are linear in $s_{i}$ and $r_{i}$. Hence, $\left\{\left(\bar{g}_{j i}\right)\right\}_{1}^{N}$ is achievable, and therefore $\mathcal{G}_{E H}$ is convex.

As a result of Lemma 8, the boundary of $\mathcal{G}_{E H}$ can be traced by solving a weighted sum throughput maximization problem. In particular, we solve for weights $w \geq 0$,

$$
\begin{aligned}
\max _{\left\{g_{1 i}, g_{2 i}\right\}} & w \sum_{i=1}^{N} g_{1 i}+\sum_{i=1}^{N} g_{2 i}, \\
\text { s.t. } & \left(\sum_{i=1}^{N} g_{1 i}, \sum_{i=1}^{N} g_{2 i}\right) \in \mathcal{G}_{E H} .
\end{aligned}
$$

By substituting (37) in (38), and separating the maximization over $\left\{s_{i}, r_{i}\right\}$ and $\left\{g_{1 i}, g_{2 i}\right\}$, the weighted sum throughput maximization problem becomes

$$
\begin{array}{lll}
\max _{\left\{s_{i}, r_{i}\right\}} & \sum_{i=1}^{N} f_{w}\left(E_{i}-s_{i}+r_{i}\right), & \\
\text { s.t. } & E_{i}-s_{i}+r_{i} \geq 0, & i=1, \ldots, N, \\
& s_{i} \geq 0, r_{i} \geq 0,(2),(3), & i=1, \ldots, N,
\end{array}
$$

where $f_{w}(p)$ is the maximum weighted sum rate function defined as

$$
f_{w}(p)=\max _{\left\{g_{1}, g_{2}\right\}} w g_{1}+g_{2} \quad \text { s.t. }\left(g_{1}, g_{2}\right) \in \mathcal{G}(p) .
$$

We next show the concavity of $f_{w}(p)$ in the following lemma 4 .

Lemma 9 The maximum weighted sum rate function $f_{w}(p)$ in (40) is concave in $p$.

Proof: Let $f_{w}(p)=w g_{1}+g_{2}$ and $f_{w}\left(p^{\prime}\right)=w g_{1}^{\prime}+g_{2}^{\prime}$, with $\left(g_{1}, g_{2}\right) \in \mathcal{G}(p)$ and $\left(g_{1}^{\prime}, g_{2}^{\prime}\right) \in \mathcal{G}\left(p^{\prime}\right)$. By Lemma 7 , we have $\left(\lambda g_{1}+(1-\lambda) g_{1}^{\prime}, \lambda g_{2}+(1-\lambda) g_{2}^{\prime}\right) \in \mathcal{G}\left(\lambda p+(1-\lambda) p^{\prime}\right)$. From the definition in (40), we can write

$$
\begin{aligned}
f_{w}\left(\lambda p+(1-\lambda) p^{\prime}\right) & \geq w\left(\lambda g_{1}+(1-\lambda) g_{1}^{\prime}\right)+\lambda g_{2}+(1-\lambda \\
& =\lambda f_{w}(p)+(1-\lambda) f_{w}\left(p^{\prime}\right),
\end{aligned}
$$

which implies the concavity of $f_{w}(p)$ in $p$.

With a concave objective (39a) and linear constraints (39b)(39c), (39) is a convex program. This problem differs from that in (20) only in the objective. Finding the respective KKT conditions, the relation between the thresholds $p_{s i}$ and $p_{r i}$, given for the single link in (15), becomes

$$
\frac{f_{w}^{\prime}\left(p_{s i}\right)}{f_{w}^{\prime}\left(p_{r i}\right)}=\eta, \quad i=1, \ldots, N
$$

\footnotetext{
${ }^{4}$ We remark that the concavity property in Lemma 9 is also shown in [17, Lemma 2] specifically for a Gaussian broadcast channel with $M \geq 2$ receivers. In fact, the weighted sum rate $f_{w}(p)$ for any setting is concave due to the possibility of time-sharing between different transmit powers. Hence, these results can be generalized to a larger class of channels.
}

where $p_{s i}$ and $p_{r i}$ are defined as the solutions to

$$
\begin{aligned}
& f_{w}^{\prime}\left(p_{s i}\right)=\eta \sum_{j=i}^{N}\left(\lambda_{j}-\beta_{j}\right), \quad i=1, \ldots, N, \\
& f_{w}^{\prime}\left(p_{r i}\right)=\sum_{j=i}^{N}\left(\lambda_{j}-\beta_{j}\right), \quad i=1, \ldots, N,
\end{aligned}
$$

and $f_{w}^{\prime}(p)$ denotes the derivative of $f_{w}(p)$ with respect to transmit power $p$. By construction, the properties in Lemmas 2, 4 and 5 extend to this case. The optimal power allocation is therefore found as in Algorithm 2 by substituting (15) with (43). The resulting thresholds satisfy (16) and Lemma 6 by construction, and therefore yield valid Lagrange multipliers through (19) and (27).

We remark that the optimal policy conforms to the doublethreshold structure defined in Theorem 1, regardless of what the weight $w$ is. However, unlike the efficient storage case in [17, Lemma 3], the power policies are no longer identical for all weights $w$. In particular, the relationship in (43) depends on the weight $w$, and hence the thresholds depleting or filling the battery in Algorithm 2 are affected by the weight. This insight applies to other channel models, and the single link, as well: in [11], [12], the optimal power policy is found to be the same for all concave power-rate functions $g(p)$. However, in the inefficient storage case, the derivative $g^{\prime}(p)$ affects how the thresholds are related, and thus the optimal policy. As a conclusion, unlike previous work with ideal batteries, the rate function plays a direct role in determining the optimal policy in the inefficient storage case.

\section{Transmission Policies with Causal Energy HARVESTING INFORMATION}

The previous sections derive optimal policies when the harvesting process over the duration of the session, i.e. $E_{i}$, $i=1, \ldots, N$, is known before the session starts. This approach provides a benchmark solution as well as insights for efficient power allocation, and is applicable in scenarios where the harvested energy is controlled or predictable [44]. For other applications where such information may not be available non-causally, in this section, we develop policies that only require causal knowledge of the harvested energy.

\section{A. Optimal Online Policy}

We refer to those transmission policies where the transmitter chooses its power value based on the energy harvested up to that point in time, i.e., with causal information, as online policies. The optimal such policy can be found by solving a dynamic program [45], which we formulate next. Let the harvested energy values $E_{i}$ and fading coefficients $h_{i}$ be i.i.d. or first order Markov processes. Such harvesting processes are considered previously in [14], [46], [47], and recent work with empirical solar and wind harvesting data confirms that a Markov process is a good model for harvested energy [48]. Finite state Markov channels are also known to be good models for Rayleigh fading channels [49]-[51].

For an energy harvesting transmitter, the states of the system at the beginning of time slot $i$ include the energy stored in the 
battery, $B_{i-1}$, history of energy harvests, $E^{i}=E_{1}, \ldots, E_{i}$, fading coefficients, $h^{i}=h_{1}, \ldots, h_{i}$, and the slot index, $i$. The node decides on its transmit power based on these variables, and hence its decision can be expressed as the action $p_{i}=$ $\phi\left(B_{i-1}, E^{i}, h^{i}, i\right)$. We remark that simultaneous storage and retrieval of energy is sub-optimal also in the online case, i.e., Lemma 1 extends to online policies. Thus, given the power policy $\left\{p_{i}\right\}_{1}^{N}$, the stored and retrieved energy values are found from (17b).

Taking the action $p_{i}=\phi\left(B_{i-1}, E^{i}, h^{i}, i\right)$, the system achieves a throughput $g\left(p_{i}, h_{i}\right)$ in time slot $i$, and leaves energy $B_{i}=B_{i-1}+\eta s_{i}-r_{i}$ in the battery for future time slots. The value function, which is the achieved throughput in time slot $i$ and the expected future throughput of the system after time slot $i$, is given by the Bellman equation,

$$
\begin{aligned}
& \max _{\phi} g\left(\phi\left(B_{i-1}, E^{i}, h^{i}, i\right), h_{i}\right) \\
&+\mathbb{E}\left[\sum_{j=i+1}^{N} g\left(\phi\left(B_{j-1}, E^{j}, h^{j}, j\right), h_{j}\right)\right] \\
&=\max _{\phi} g\left(\phi\left(B_{i-1}, E^{i}, h^{i}, i\right), h_{i}\right) \\
&+\mathbb{E}\left[V\left(B_{i}, E^{i+1}, h^{i+1}, i+1\right)\right],
\end{aligned}
$$

where the expectations are taken over the distribution of the harvesting process $E_{i}$ and fading process $h_{i}$. The optimal online power policy $\phi^{*}($.$) is the maximizer of the Bellman$ equation in (45) [45]. Note that the dimension of the optimization variable in (45) increases with $i$. Thus, solving this problem through value iteration has exponential complexity, and is intractable for large $N$. However, it is possible to simplify the problem by observing the Markovian property of the harvesting and channel fading processes. In particular, only $B_{i-1}$ and $E_{i}$ impose a constraint on the transmit power, and only $h_{i}$ affects the rate, in time slot $i$. Since $E_{i}$ and $h_{i}$ are i.i.d or Markovian, future realizations of these variables are independent of the past values given their current values. Hence, having different actions for different values of $\left\{E_{i}\right\}_{1}^{i-1}$ and $\left\{h_{i}\right\}_{1}^{i-1}$ does not affect the value function, and therefore we can simplify the actions as $\phi\left(B_{i-1}, E^{i}, h^{i}, i\right)=$ $\phi\left(B_{i-1}, E_{i}, h_{i}, i\right)$. This yields the simplified Bellman equation for i.i.d. arrivals and fading,

$$
\begin{aligned}
V\left(B_{i-1}, E_{i}, h_{i}, i\right)= & \max _{\phi} g\left(\phi\left(B_{i-1}, E_{i}, h_{i}, i\right), h_{i}\right) \\
& +\mathbb{E}\left[V\left(B_{i}, E_{i+1}, h_{i+1}, i+1\right)\right],
\end{aligned}
$$

which can be easily computed using value iteration. Namely, starting from $i=N$ and choosing $V\left(B_{N-1}, E_{N}, h_{N}, N+\right.$ $1)=0$, optimal actions $\phi\left(B_{N-1}, E_{N}, h_{N}, N\right)$ and value functions $V\left(B_{N-1}, E_{N}, h_{N}, N\right)$ are calculated. These values are then used to calculate the optimal actions and value functions at $i=N-1$ from (46), and the process is repeated for all $i$.

Finally, we consider an infinite-horizon problem, i.e., $N \rightarrow$ $\infty$, and find the optimal online policy using a discounted problem. This is also the policy that the value iteration algorithm on (46) converges to for very large $N$. To find this policy, denoted by $\phi^{*}\left(B_{i-1}, E_{i}, h_{i}\right)$, we introduce a discount

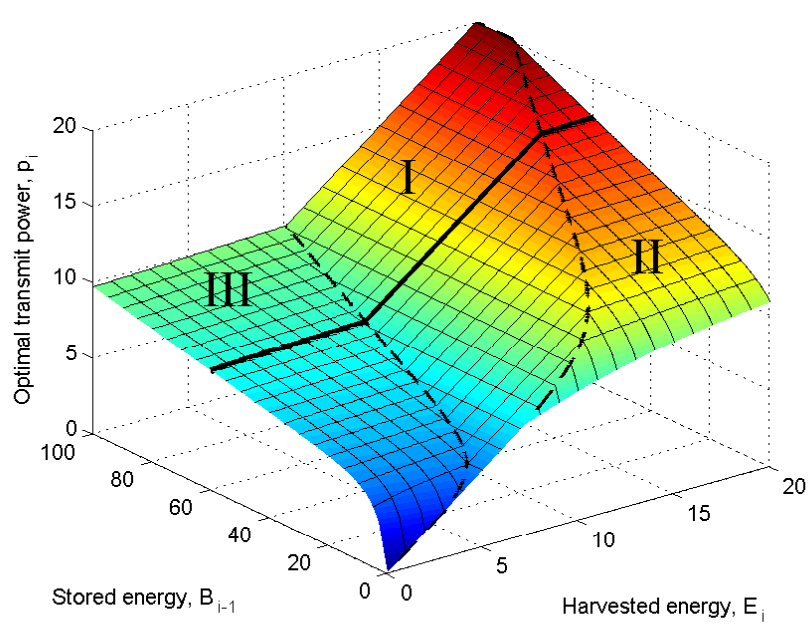

Fig. 5. Optimal online transmission power for i.i.d. energy arrivals.

factor $\beta \leq 1$ and write the Bellman equation as

$$
\begin{aligned}
V\left(B_{i-1}, E_{i}, h_{i}\right)= & \max _{\phi} g\left(\phi\left(B_{i-1}, E_{i}, h_{i}\right), h_{i}\right) \\
& +\beta \mathbb{E}\left[V\left(B_{i}, E_{i+1}, h_{i+1}\right)\right] .
\end{aligned}
$$

Starting with an arbitrary set of initial actions, iterating (47) converges to the optimal policy $\phi^{*}\left(B_{i-1}, E_{i}, h_{i}\right)$. As the discount factor $\beta \rightarrow 1$, the resulting policy approaches the optimal infinite horizon policy.

As an example, Fig. 5 shows the optimal infinite horizon policy for a non-fading Gaussian channel with $h_{i}=1$, a finitesized battery $E_{\max }=100$, and i.i.d. uniform energy harvests in $[0,20]$. Note that for a fixed stored energy, the optimal online policy exhibits a double-threshold structure similar to that in Theorem 1, e.g., the bold line for $B_{i-1}=60$ in the figure. The optimal transmit power is equal to the harvested energy for a range of $B_{i-1}$ and $E_{i}$ values, marked as region I. Regions II and III are separated from region I by a set of thresholds, indicated with dashed lines. Within these regions, the transmit powers vary only slightly with harvested energy rate $E_{i}$. The two thresholds separating region I from regions II and III are observed to satisfy the relationship in (15) for each $B_{i-1}$. The thresholds, however, change with $B_{i-1}$ : For small $B_{i-1}$, the thresholds are lower, with $p_{r i}=0$ at $B_{i-1}=0$ since retrieving energy is not feasible. For large $B_{i-1}$, the thresholds are higher, reaching to $p_{s i}>20 \mathrm{~mW}$ at $B_{i-1}=E_{\max }$, since storing energy is not feasible.

For harvesting processes with memory, we consider two scenarios with Markovian energy harvests in Fig. 6 and Fig. 7. In Fig. 6, harvesting is a bursty process where the next energy harvest remains the same, i.e., $E_{i+1}=E_{i}$, with probability 0.5 , and a new value that is uniform in $[0,20]$ is generated with probability 0.5 . Hence, the process consists of bursts of constant rate harvests. As seen in Fig. 6, this harvesting model also yields a double-threshold policy that resembles the i.i.d. case in Fig. 5. In Fig. 7, harvested energy $E_{i}$ performs a random walk on $[0,20]$, where it increases or decreases 1 unit with probability 0.4 each, and remains the 


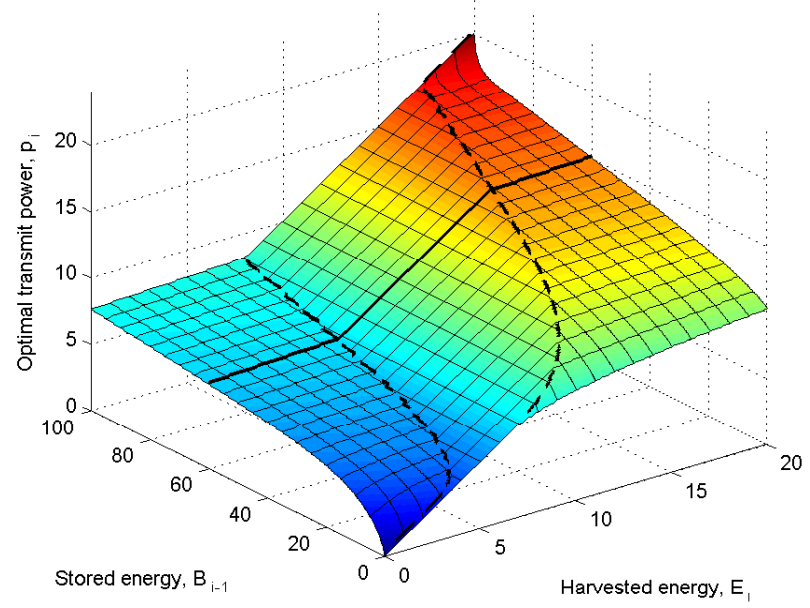

Fig. 6. Optimal online transmission power for the bursty energy harvesting model.

same with probability 0.2 . In this case, the optimal policy is to consume all harvested energy, which does not show a threshold characteristic. This is intuitive, because a high or low harvest rate is sustained for extended periods in this model, and consistently storing or retrieving would likely overflow or deplete the battery.

\section{B. Proposed Online Policy}

Sections III-VI show that the optimal policy has a doublethreshold structure where the thresholds are related for all $i=$ $1, \ldots, N$. The infinite-horizon optimal online policy found in Section VII-A for i.i.d. arrivals also exhibits a similar doublethreshold structure. With these in mind, in this section, we propose a simpler online double-threshold policy by assigning constant thresholds throughout the communication session.

We first consider a non-fading channel and Markovian harvested energy values $E_{i}$ with stationary probability distribution $f_{E}(E)$. We propose finding fixed thresholds $p_{s i}=p_{s}$ and $p_{r i}=p_{r}, i=1, \ldots, N$, that satisfy (15) and

$$
\eta \int_{p_{s}}^{\infty}\left(e-p_{s}\right) f_{E}(e) d e-\int_{0}^{p_{r}}\left(p_{r}-e\right) f_{E}(e) d e=0 .
$$

This equation can be interpreted as an energy stability condition, since it ensures that the expected energy stored in and retrieved from the battery are equal. Thus, neither the energy storage is underutilized, nor an excessive amount of energy is stored without utility. Note that at $\eta=1$, this reduces to a constant power policy that preserves energy-neutrality, and resembles the best-effort transmission scheme of [52] which is optimal for infinite length transmission. On the other hand, at $\eta=0$, (48) is only satisfied with $p_{r}=0$ and $p_{s} \rightarrow \infty$. This means that no energy is stored, i.e., $p_{i}=E_{i}$, which is optimal since $\eta=0$.

Above policy satisfying (48) can be readily extended to a fading channel with Markovian channel coefficients $h_{i}$ and joint stationary distribution $f_{E, H}(e, h)$ by finding water level

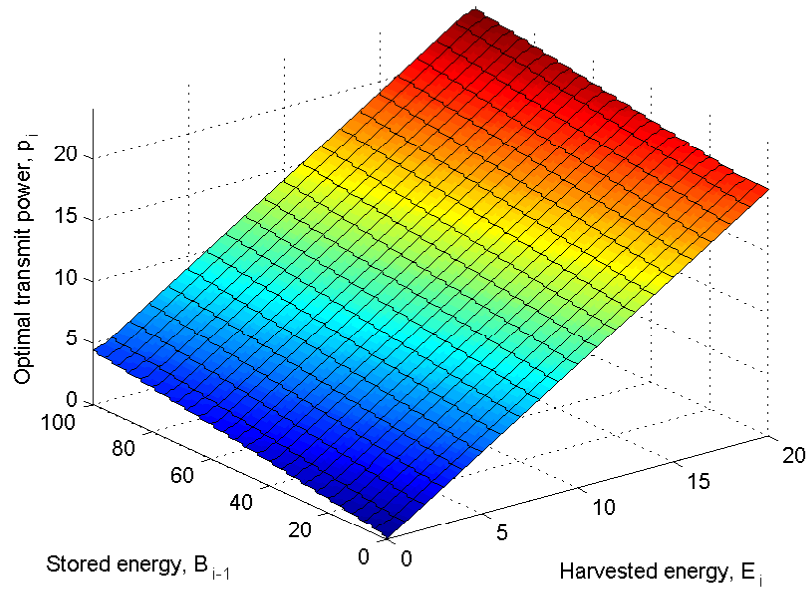

Fig. 7. Optimal online transmission power for the random walk energy harvesting model.

thresholds $v_{s i}=v_{s}$ and $v_{r i}=v_{r}, i=1, \ldots, N$, that satisfy (32) and

$$
\begin{aligned}
\iint_{0}^{\infty} \eta & {\left[e-\left[v_{s}-\frac{1}{h}\right]^{+}\right]^{+} } \\
& -\left[v_{r}-\frac{1}{h}-e\right]^{+} f_{E, H}(e, h) d e d h=0
\end{aligned}
$$

where (49) is the fading equivalent of the energy stability condition in (48).

\section{NUMERICAL RESULTS}

In this section, we provide numerical results on the performances of the optimal offline policy and the online policies. We simulate communication sessions consisting of $N=10^{4}$ time slots, with a slot length of $\tau=10 \mathrm{~ms}$. Since the model in Section II assumed unit slot length, the optimal policies in this case are found by scaling transmit powers and consumed energy values accordingly. We consider an energy harvesting transmitter node equipped with a battery of size $1 \mathrm{~mJ}$ and initial charge $E_{0}^{b a t}=0$. We have the Gaussian noise spectral density of $N_{0}=10^{-19} \mathrm{~W} / \mathrm{Hz}$ at the receiver, and a bandwidth of $1 \mathrm{MHz}$. The path loss between the transmitter and receiver is $h=-100 \mathrm{~dB}$.

For the purpose of comparison, we introduce two algorithms. The first is the directional water-filling $(D W F)$ algorithm of [13], which is indifferent to the storage efficiency $\eta$, and a feasible policy is obtained as in [13]. The second is the efficiency-adaptive directional water-filling algorithm, which is obtained by forcing the two thresholds of the optimal offline algorithm in Section III to be equal, thus resembling DWF in [13]. However, it accounts for the storage efficiency $\eta$ when choosing its constant water levels, and therefore is a near-optimal heuristic.

We consider the single-user setting with a finite-sized battery in Section IV. Fig. 8 shows the throughput for the offline and online policies versus storage efficiency $\eta$ when the harvested energy $E_{i}$ at each time slot of length $10 \mathrm{~ms}$ is generated in an i.i.d. fashion, distributed uniformly in 


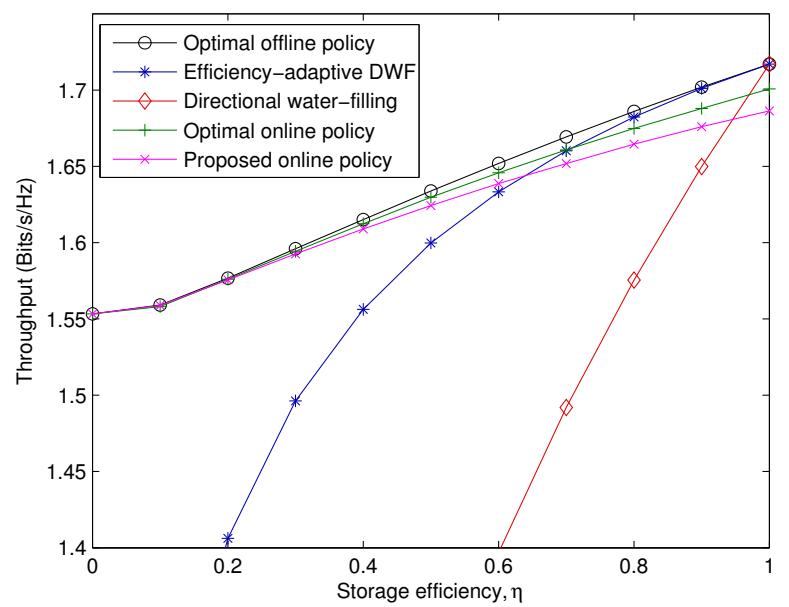

Fig. 8. Throughput for a static channel with i.i.d. energy arrivals and an average harvesting rate of $10 \mathrm{~mW}$.

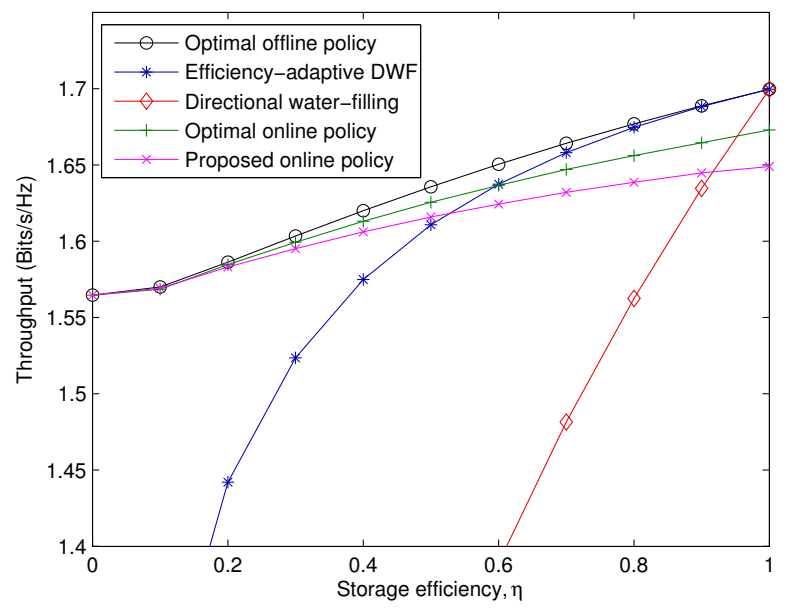

Fig. 9. Throughput for a static channel with Markov (bursty) energy arrivals and an average harvesting rate of $10 \mathrm{~mW}$.

$[0,200] \mu \mathrm{J}$. This corresponds to an average energy harvesting rate of $10 \mathrm{~mW}$. Simulations are repeated for bursty and random walk arrival models of Section VII-A in Fig. 9 and Fig. 10, respectively, for a harvested energy range of $[0,200] \mu \mathrm{J}$. We observe that the performance of DWF degrades rapidly with decreasing $\eta$, since it does not adapt to storage efficiency. Efficiency-adaptive DWF performs reasonably well for high storage efficiency, but worse at low storage efficiency since it also relies on frequently storing and retrieving energy. Moreover, in all cases, the proposed online policy performs very close to the optimal online policy, both providing a notable improvement over DWF and efficiency-adaptive DWF.

In Fig. 11, we compare the throughput of offline and online policies for a fading channel. We consider Rayleigh fading with $\mathbb{E}\left[h_{i}\right]=-100 \mathrm{~dB}$, and the remaining parameters are unchanged from those in Fig. 8. Here, the optimal offline and online policies compare similar to the non-fading case. We observe that efficiency-adaptive DWF performs close to optimal for high storage efficiency, while DWF rapidly departs

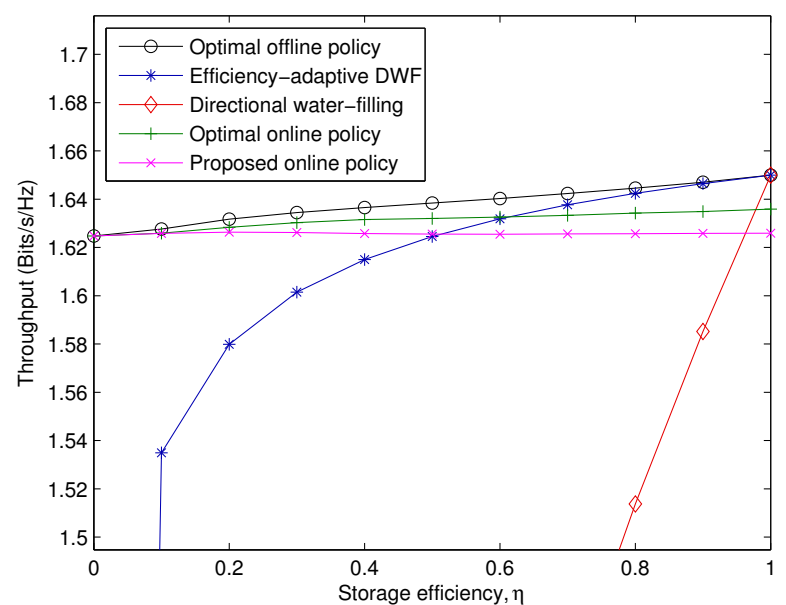

Fig. 10. Throughput for a static channel with Markov (random walk) energy arrivals and an average harvesting rate of $10 \mathrm{~mW}$.

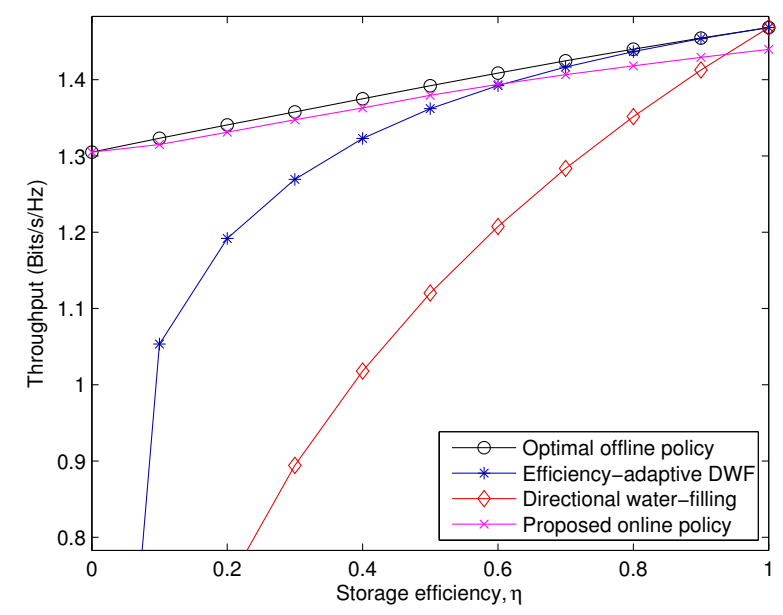

Fig. 11. Throughput for a Rayleigh fading channel with i.i.d. energy arrivals and an average harvesting rate of $10 \mathrm{~mW}$.

from the optimal as $\eta$ decreases. The proposed online policy is notably close to the optimal for all storage efficiency values.

We next consider an average harvesting rate of $80 \mu \mathrm{W}$, which is more realistic for small-sized energy harvesting sensor nodes with limited access to ambient energy. We generate energy arrivals accordingly, while the remaining parameters are unchanged. Figs. 12-15 present the throughput for the offline and online policies versus storage efficiency $\eta$ for i.i.d., bursty, and random walk energy harvests in a static channel, and i.i.d. energy harvests in a Rayleigh fading channel, respectively. The significance of the double-threshold policy is more pronounced in this low-power scenario, as the performance of both DWF and efficiency-adaptive DWF quickly depart from that of the optimal as $\eta$ decreases.

We plot the throughput of the above policies relative to the optimal offline policy, i.e., scaled by the optimal throughput, as a function of average energy harvesting rate in Fig. 16, for i.i.d. energy harvests in a static channel with the same parameters. We observe that while the performance of the optimal online and proposed online algorithms are virtually identical to that 


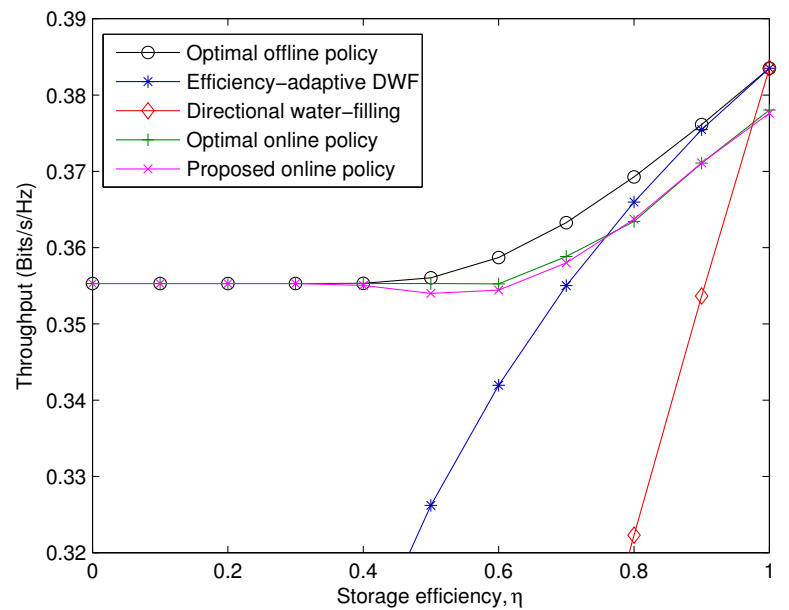

Fig. 12. Throughput for a static channel with i.i.d. energy arrivals and an average harvesting rate of $80 \mu \mathrm{W}$.

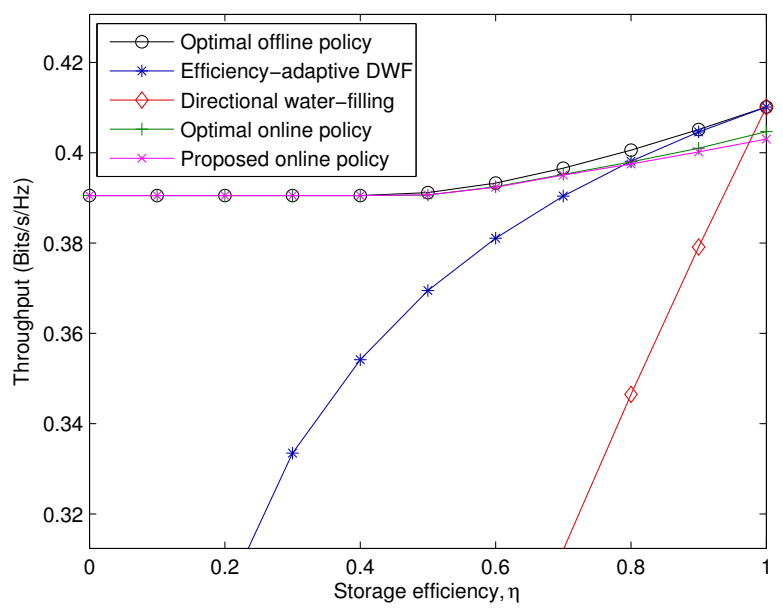

Fig. 13. Throughput for a static channel with Markov (bursty) energy arrivals and an average harvesting rate of $80 \mu \mathrm{W}$.

of the optimal offline policy, the performance of efficiencyadaptive DWF and DWF falls to as low as $90 \%$ and $67 \%$ of the optimal throughput, respectively, at low harvesting rates.

Finally, to examine the dynamics of the policies further, we present a smaller numerical example with $N=5$ time slots of duration $10 \mathrm{~ms}$, storage efficiency $\eta=0.66$, battery capacity $E_{\max }=20 \mu \mathrm{J}$, and energy harvests $\left[\begin{array}{lllll}18 & 20 & 2 & 9 & 4\end{array}\right] \mu \mathrm{J}$. In this scenario, the optimal power policy sets thresholds $p_{s}=1.43 \mathrm{~mW}$ and $p_{r}=0.61 \mathrm{~mW}$, and yields the transmit powers $\mathbf{p}=\left[\begin{array}{lllll}1.43 & 1.43 & 0.61 & 0.90 & 0.61\end{array}\right] \mathrm{mW}$ with an average throughput of $0.4861 \mathrm{bits} / \mathrm{sec} / \mathrm{Hz}$. In comparison, efficiencyadaptive DWF yields $\mathbf{p}=\left[\begin{array}{lllll}0.93 & 0.93 & 0.93 & 0.93 & 0.93\end{array}\right] \mathrm{mW}$ with average throughput $0.4733 \mathrm{bits} / \mathrm{sec} / \mathrm{Hz}$, and DWF yields $\mathbf{p}=\left[\begin{array}{lllll}0.70 & 0.70 & 0.70 & 0.70 & 0.70\end{array}\right] \mathrm{mW}$ with average throughput $0.3825 \mathrm{bits} / \mathrm{sec} / \mathrm{Hz}$. Note that the optimal offline policy consumes more energy in the first two time slots, and does not insist on equalizing the powers $p_{2}$ and $p_{3}$. This is to its benefit, because for these transmit powers, energy storage loss overcomes the advantage of constant power transmis-

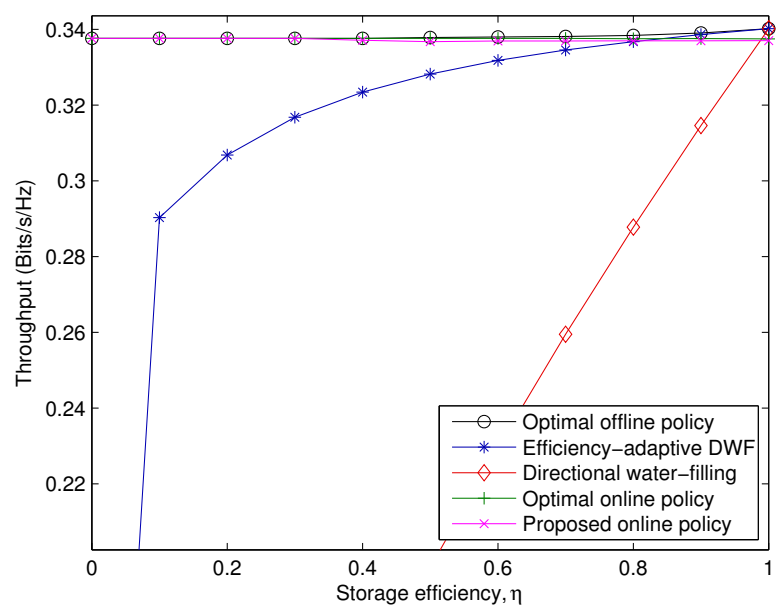

Fig. 14. Throughput for a static channel with Markov (random walk) energy arrivals and an average harvesting rate of $80 \mu \mathrm{W}$.

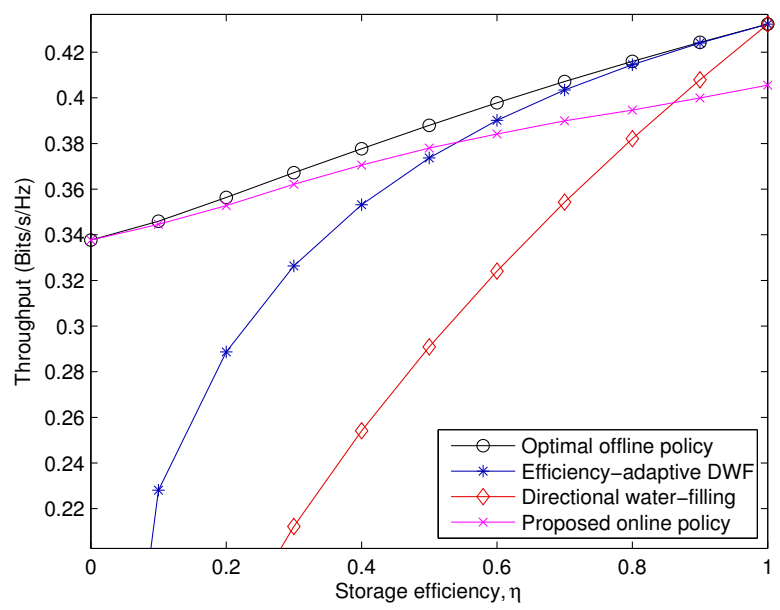

Fig. 15. Throughput for a Rayleigh fading channel with i.i.d. energy arrivals and an average harvesting rate of $80 \mu \mathrm{W}$.

sion. Meanwhile, being aware of the storage inefficiency, the efficiency-adaptive DWF algorithm chooses a constant transmit power of $0.93 \mathrm{~mW}$, while the DWF algorithm chooses a constant transmit power of $0.70 \mathrm{~mW}$. As a result, the average throughput of the optimal offline algorithm is significantly better than that of DWF, and is only approached by efficiencyadaptive DWF.

\section{CONCLUSion}

We identify the throughput optimal transmit power policy for an energy harvesting transmitter with a battery that has storage inefficiency. We show that the optimal policy has a double-threshold structure, where the thresholds are determined by the energy harvesting process and the storage efficiency. We show that the thresholds are constant if battery is not completely empty or full, and the thresholds only increase when the battery is empty, and only decrease when the battery is full. We develop an algorithm to find the optimum thresholds. We extend the solution to a fading channel, and 


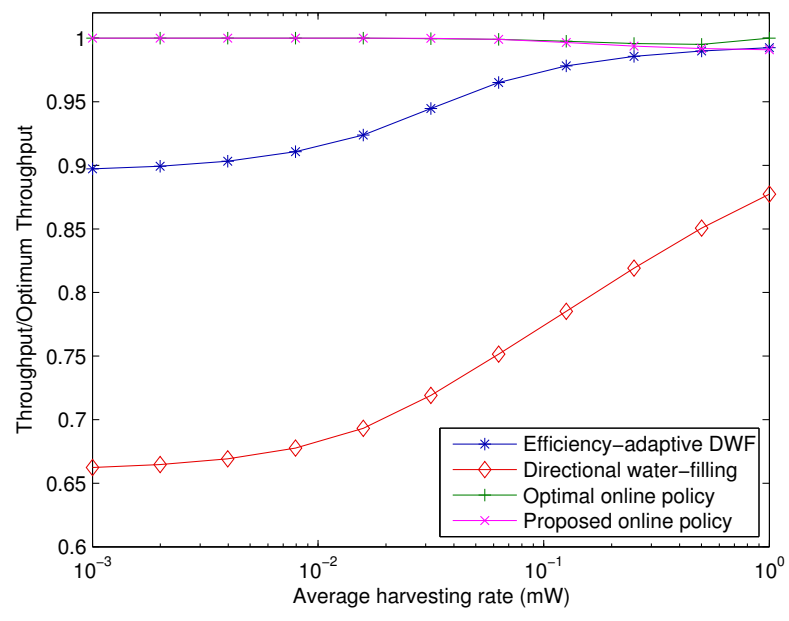

Fig. 16. Throughput of efficiency-adaptive DWF, DWF, and online policies relative to optimal throughput.

develop a directional water-filling algorithm with a doublethreshold policy acting on water levels. In the broadcast channel, we obtain throughput regions by maximizing weighted sum throughput for all weights, where the optimal policy in each case is a double-threshold policy. Finally, we use a dynamic programming formulation to develop an optimum online algorithm, and numerically observe that it follows a similar double-threshold structure. We further propose a simpler online double-threshold policy with low complexity, and observe experimentally that it performs close to its optimal counterpart. An insight drawn from these results is that when battery inefficiency is taken into consideration, the optimal power policy is no longer piecewise constant as was the case in previous work with ideal batteries. Instead, two thresholds emerge in both the offline and online optimal policies, between which harvested energy is consumed immediately, i.e., without energy storage or retrieval. When battery is set to be lossless, these two thresholds are equal, and the policies converge to previous results. In essence, double-threshold policies result from the inefficiency of the battery, and introduce an interval within which the losses due to inefficiency outweigh the benefits of storage. In addition, we observe that the conventional directional water-filling algorithm, which does not adapt to storage inefficiency, incurs a significant performance loss as storage efficiency decreases. Important future directions are incorporating other practical aspects of physical systems such as processing power and communication overheads to our model, leading to practical implementation of these communication systems. Finally, the results of this paper where we have investigated charging/discharging imperfections that occur at the time of energy saving/retrieval should further be combined with other types of battery imperfections such as battery degradation and energy leakage that occur over time.

\section{REFERENCES}

[1] A. J. Goldsmith and S. B. Wicker. Design challenges for energyconstrained ad hoc wireless networks. IEEE Wireless Comm., 9(4):8-27, 2002.
[2] A. Ephremides. Energy concerns in wireless networks. IEEE Wireless Comm., 9(4):48-59, 2002.

[3] E. Uysal-Biyikoglu, B. Prabhakar, and A. El Gamal. Energy-efficient packet transmission over a wireless link. IEEE/ACM Trans. on Networking, 10(4):487-499, August 2002.

[4] E. Uysal-Biyikoglu and A. El Gamal. On adaptive transmission for energy efficiency in wireless data networks. IEEE Trans. on Inform. Theory, 50(12):3081-3094, 2004.

[5] M. Zorzi and R. R. Rao. Energy-constrained error control for wireless channels. IEEE Personal Comm., 4(6):27-33, 1997.

[6] S. Cui, A. J. Goldsmith, and A. Bahai. Energy-constrained modulation optimization. IEEE Trans. on Wireless Comm., 4(5):2349-2360, 2005.

[7] M. A. Zafer and E. Modiano. A calculus approach to energy-efficient data transmission with quality-of-service constraints. IEEE/ACM Trans. on Networking, 17(3):898-911, 2009.

[8] M. A. Zafer and E. Modiano. Optimal rate control for delay-constrained data transmission over a wireless channel. IEEE Trans. on Inform. Theory, 54(9):4020-4039, 2008.

[9] M. J. Neely. Energy optimal control for time-varying wireless networks. IEEE Trans. on Inform. Theory, 52(7):2915-2934, 2006.

[10] M. J. Neely. Optimal energy and delay tradeoffs for multiuser wireless downlinks. IEEE Trans. on Inform. Theory, 53(9):3095-3113, 2007.

[11] J. Yang and S. Ulukus. Optimal packet scheduling in an energy harvesting communication system. IEEE Trans. on Comm., 60(1):220230, January 2012.

[12] K. Tutuncuoglu and A. Yener. Optimum transmission policies for battery limited energy harvesting nodes. IEEE Trans. on Wireless Comm., 11(3):1180-1189, March 2012.

[13] O. Ozel, K. Tutuncuoglu, J. Yang, S. Ulukus, and A. Yener. Transmission with energy harvesting nodes in fading wireless channels: Optimal policies. IEEE Jour. on Selected Areas in Comm., 29(8):1732-1743, September 2011.

[14] C. K. Ho and R. Zhang. Optimal energy allocation for wireless communications with energy harvesting constraints. IEEE Trans. on Signal Processing, 60(9):4808-4818, September 2012.

[15] J. Yang, O. Ozel, and S. Ulukus. Broadcasting with an energy harvesting rechargeable transmitter. IEEE Trans. on Wireless Comm., 11(2):571583, February 2012.

[16] M. A. Antepli, E. Uysal-Biyikoglu, and H. Erkal. Optimal packet scheduling on an energy harvesting broadcast link. IEEE Jour. on Selected Areas in Comm., 29(8):1721-1731, September 2011.

[17] O. Ozel, J. Yang, and S. Ulukus. Optimal broadcast scheduling for an energy harvesting rechargeable transmitter with a finite capacity battery. IEEE Trans. on Wireless Comm., 11(6):2193-2203, June 2012.

[18] J. Yang and S. Ulukus. Optimal packet scheduling in a multiple access channel with energy harvesting transmitters. Jour. of Comm. and Networks, Special Issue on Energy Harvesting in Wireless Networks, 14(2):140-150, April 2012.

[19] K. Tutuncuoglu and A. Yener. Sum-rate optimal power policies for energy harvesting transmitters in an interference channel. Jour. of Comm. and Networks, Special Issue on Energy Harvesting in Wireless Networks, 14(2):151-161, April 2012.

[20] D. Gunduz and B. Devillers. Two-hop communication with energy harvesting. In IEEE CAMSAP, December 2011.

[21] C. Huang, R. Zhang, and S. Cui. Throughput maximization for the Gaussian relay channel with energy harvesting constraints. IEEE Jour. on Selected Areas in Comm., 31(8):1469-1479, August 2013.

[22] O. Orhan and E. Erkip. Optimal transmission policies for energy harvesting two-hop networks. In CISS, March 2012.

[23] O. Orhan and E. Erkip. Throughput maximization for energy harvesting two-hop networks. In IEEE ISIT, July 2013.

[24] I. Ahmed, A. Ikhlef, R. Schober, and R. K. Mallik. Power allocation for conventional and buffer-aided link adaptive relaying systems with energy harvesting nodes. IEEE Trans. on Wireless Comm., 13(3):1182-1195, March 2014.

[25] I. Ahmed, A. Ikhlef, R. Schober, and R. K. Mallik. Joint power allocation and relay selection in energy harvesting $\mathrm{AF}$ relay systems. IEEE Wireless Comm. Letters, 2(2):239-242, April 2013.

[26] Y. Luo, J. Zhang, and K. B. Letaief. Optimal scheduling and power allocation for two-hop energy harvesting communication systems. IEEE Trans. on Wireless Comm., 12(9):4729-4741, September 2013.

[27] O. Orhan, D. Gunduz, and E. Erkip. Throughput maximization for an energy harvesting communication system with processing cost. In IEEE ITW, September 2012.

[28] J. Xu and R. Zhang. Throughput optimal policies for energy harvesting wireless transmitters with non-ideal circuit power. IEEE Jour. on Selected Areas in Comm., 32(2):322-332, February 2014. 
[29] M. Gregori and M. Payaro. Throughput maximization for a wireless energy harvesting node considering the circuitry power consumption. In IEEE VTC Fall, September 2012.

[30] Q. Bai and J. A. Nossek. Throughput maximization for energy harvesting nodes with generalized circuit power modelling. In IEEE SPAWC, June 2012.

[31] B. Gurakan, O. Ozel, J. Yang, and S. Ulukus. Energy cooperation in energy harvesting communications. IEEE Trans. on Comm., 61(12):48844898, December 2013.

[32] K. Tutuncuoglu and A. Yener. Multiple access and two-way channels with energy harvesting and bidirectional energy cooperation. In UCSD ITA, February 2013.

[33] K. Tutuncuoglu and A. Yener. Cooperative energy harvesting communications with relaying and energy sharing. In IEEE ITW, September 2013.

[34] H. Mahdavi-Doost and R. D. Yates. Energy harvesting receivers: Finite battery capacity. In IEEE ISIT, July 2013.

[35] K. Tutuncuoglu and A. Yener. Communicating with energy harvesting transmitters and receivers. In UCSD ITA, February 2012.

[36] S. Sudevalayam and P. Kulkarni. Energy harvesting sensor nodes: Survey and implications. IEEE Comm. Surveys \& Tutorials, 13(3):443-461, September 2011.

[37] J. Taneja, J. Jeong, and D. Culler. Design, modeling, and capacity planning for micro-solar power sensor networks. In IPSN, April 2008.

[38] B. Devillers and D. Gunduz. A general framework for the optimization of energy harvesting communication systems with battery imperfections. Jour. of Comm. and Networks, Special Issue on Energy Harvesting in Wireless Networks, 14(2):130-139, April 2012.

[39] H. Ibrahim, A. Ilinca, and J. Perron. Energy storage systems characteristics and comparisons. Renewable and Sustainable Energy Reviews, 12(5):1221-1250, June 2008.

[40] I. Hadjipaschalis, A. Poullikkas, and V. Efthimiou. Overview of current and future energy storage technologies for electric power applications. Renewable and Sustainable Energy Reviews, 13(6):1513-1522, August 2009.

[41] S. Reddy and C. R. Murthy. Duty cycling and power management with a network of energy harvesting sensors. In IEEE CAMSAP, December 2011.

[42] S. Reddy and C. R. Murthy. Dual-stage power management algorithms for energy harvesting sensors. IEEE Trans. on Wireless Comm. 11(4):1434 -1445, April 2012.

[43] T. M. Cover and J. A. Thomas. Elements of Information Theory. John Wiley \& Sons, New Jersey, 2006.

[44] M. Gorlatova, A. Wallwater, and G. Zussman. Networking low-power energy harvesting devices: Measurements and algorithms. In IEEE INFOCOM, April 2011.

[45] D. P. Bertsekas. Dynamic programming and optimal control. Athena Scientific, Belmont, MA, 1995.

[46] B. Medepally, N. B. Mehta, and C. R. Murthy. Implications of energy profile and storage on energy harvesting sensor link performance. In IEEE GLOBECOM, November 2009.

[47] J. Lei, R. D. Yates, and L. Greenstein. A generic model for optimizing single-hop transmission policy of replenishable sensors. IEEE Trans. on Wireless Comm., 8(2):547-551, February 2009.

[48] C. K. Ho, P. D. Khoa, and P. C. Ming. Markovian models for harvested energy in wireless communications. In IEEE ICCS, November 2010.

[49] H. S. Wang and N. Moayeri. Finite-state Markov channel - a usefu model for radio communication channels. IEEE Trans. on Vehicular Tech., 44(1):163-171, February 1995.

[50] H. S. Wang and P. Chang. On verifying the first-order Markovian assumption for a Rayleigh fading channel model. IEEE Trans. on Vehicular Tech., 45(2):353-357, May 1996.

[51] Q. Zhang and S. A. Kassam. Finite-state Markov model for Rayleigh fading channels. IEEE Trans. on Comm., 47(11):1688-1692, November 1999.

[52] O. Ozel and S. Ulukus. Achieving AWGN capacity under stochastic energy harvesting. IEEE Trans. on Information Theory, 58(10):64716483, October 2012.

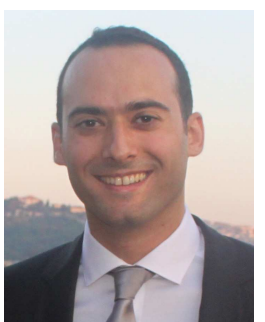

Kaya Tutuncuoglu (S'08) received his two B.S. degrees in Electrical and Electronics Engineering and in Physics from the Middle East Technical University, Ankara, Turkey, in 2008 and 2009, respectively. He is currently pursuing the Ph.D. degree and has been a graduate research assistant with the Department of Electrical Engineering, Pennsylvania State University, University Park, PA, since 2009. His research interests include green communications, energy harvesting, and resource allocation for wireless networks. He received the AT\&T Graduate Fellowship at Penn State in 2012, the IEEE Marconi Prize Paper Award in Wireless Communications in 2014, and the Leonard A. Doggett Award for Outstanding Writing in Electrical Engineering at Penn State in 2014.

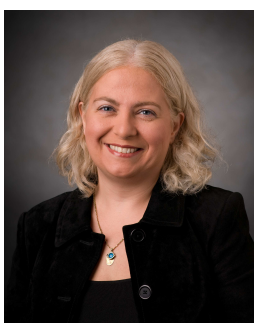

Aylin Yener (S'91-M'00-SM'13-F'14) received the B.Sc. degree in electrical and electronics engineering, and the B.Sc. degree in physics, from Bogazici University, Istanbul, Turkey; and the M.S. and Ph.D. degrees in electrical and computer engineering from Wireless Information Network Laboratory (WINLAB), Rutgers University, New Brunswick, NJ. She is a professor of Electrical Engineering at The Pennsylvania State University, University Park, PA since 2010, where she joined the faculty as an assistant professor in 2002. During the academic year 2008-2009, she was a Visiting Associate Professor with the Department of Electrical Engineering, Stanford University, CA. Her research interests are in information theory, communication theory and network science with recent emphasis on green communications and information security.

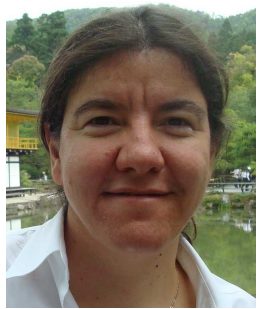

Sennur Ulukus (S'90-M'98) is a Professor of Electrical and Computer Engineering at the University of Maryland at College Park, where she also holds a joint appointment with the Institute for Systems Research (ISR). Prior to joining UMD, she was a Senior Technical Staff Member at AT\&T LabsResearch. She received her Ph.D. degree in Electrical and Computer Engineering from Wireless Information Network Laboratory (WINLAB), Rutgers University, and B.S. and M.S. degrees in Electrical and Electronics Engineering from Bilkent University. Her research interests are in wireless communication theory and networking, network information theory for wireless communications, signal processing for wireless communications, information theoretic physical layer security, and energy harvesting communications.

Dr. Ulukus received the 2003 IEEE Marconi Prize Paper Award in Wireless Communications, an 2005 NSF CAREER Award, the 2010-2011 ISR Outstanding Systems Engineering Faculty Award, and the 2012 George Corcoran Education Award. She served as an Associate Editor for the IEEE Transactions on Information Theory (2007-2010) and IEEE Transactions on Communications (2003-2007). She served as a Guest Editor for the IEEE Journal on Selected Areas in Communications for the special issue on wireless communications powered by energy harvesting and wireless energy transfer (2015), Journal of Communications and Networks for the special issue on energy harvesting in wireless networks (2012), IEEE Transactions on Information Theory for the special issue on interference networks (2011), IEEE Journal on Selected Areas in Communications for the special issue on multiuser detection for advanced communication systems and networks (2008). She served as the TPC co-chair of the 2014 IEEE PIMRC, Communication Theory Symposium at 2014 IEEE Globecom, Communication Theory Symposium at 2013 IEEE ICC, Physical-Layer Security Workshop at 2011 IEEE Globecom, Physical-Layer Security Workshop at 2011 IEEE ICC, 2011 Communication Theory Workshop (IEEE CTW), Wireless Communications Symposium at 2010 IEEE ICC, Medium Access Control Track at 2008 IEEE WCNC, and Communication Theory Symposium at 2007 IEEE Globecom. She was the Secretary of the IEEE Communication Theory Technical Committee (CTTC) in 2007-2009. 